\title{
Macro- and micro-nutrient-based multiplex stress conditions modulate in vitro tumorigenesis and aggressive behavior of breast cancer spheroids
}

\author{
Sukanya Gayan ${ }^{1} \cdot$ Abhishek Teli $^{1} \cdot$ Anish Nair ${ }^{1} \cdot$ Geetanjali Tomar $^{1} \cdot$ Tuli Dey $^{1}$ (i)
}

Received: 7 August 2021 / Revised: 30 October 2021 / Accepted: 10 November 2021 / Published online: 25 November 2021

(c) The Author(s), under exclusive licence to Springer Nature Switzerland AG 2021

\begin{abstract}
Purpose The aggressive nature of a tumor is presumably its inherent one, but different environmental cues can manipulate it in many ways. In this context, the influence of metabolic stresses on tumor behavior needs to be analyzed to understand their far-reaching implications on tumor aggression and dormancy. This work investigates different facets of the tumor, such as tumorigenic capacity, tumor phenotype, and migration, under multiple metabolic stress conditions.

Methods Non-invasive and invasive multicellular spheroids (MTS) were created and subjected to multiple stress conditions, namely glucose, amino acid, and oxygen deprivation. Altered behavior of the MTS has been evaluated in the context of in vitro tumorigenesis, spheroid formation capacity, phenotype, mRNA profile, migration, and recruitment of mesenchymal stem cells.

Results The metabolic stress conditions were observed to negatively impact the in vitro tumorigenesis and spheroid formation process of invasive and non-invasive breast cancer cells. While the stress seemingly influences the growth and phenotype of spheroids, it does not alter the organization of sub-cellular entities significantly. Metabolic stress conditions impact the transcriptomic landscape of hypoxic, angiogenic, ECM deformation, glycolysis shift, and protein starvation-related gene clusters. MTSs do not adhere or migrate under stress, but they exhibit different modalities of migration when rescued. Invasive spheroids, after the rescue, exhibit increased aggressiveness. Furthermore, stressed spheroid was observed to control the migration and recruitment of mesenchymal stem cells.

Conclusion Multiplex metabolic stresses could control the tumorigenesis while influencing the physiology of invasive and non-invasive breast cancer spheroids along with their migration pattern and tumor-stromal crosstalk.
\end{abstract}

Keywords Multicellular spheroid $\cdot$ Nutrient stress $\cdot$ Hypoxia $\cdot$ Multiple stresses $\cdot$ Metastasis $\cdot$ Dormancy $\cdot$ Mesenchymal stem cell

\section{Introduction}

Cancer is one of the fatal diseases due to its aggressive behavior. A growing tumor ( $>2-5 \mathrm{~mm}$ diameter) with a necrotic core and a proliferating periphery initiates the shedding of metastatic cells and instigates the growth of occult tumors in other tissues [1]. The migration of tumor cells towards the future metastatic niche is a complex process. Investigation of this process has garnered much interest in

Tuli Dey

tulidey@unipune.ac.in

1 Institute of Bioinformatics \& Biotechnology, Savitribai Phule Pune University, Pune, India recent times due to its potential to provide much deeper insight into the tumor's social intelligence [2] and potential cure. Many factors such as tumor microenvironment, extracellular matrix, and environmental stress are presumed to influence tumor behaviors, including metastasis [3, 4]. Also, inter-cellular stress conditions like deprivation of nutrition and oxygen are assumed to play an influential role in angiogenesis and aggression of the tumor [5-7]. Rapidly growing tumors utilize the available glucose and initiate acidosis [8, 9], creating oxygen scarcity and hypoxic stress. As observed, metabolic stress inducts angiogenesis in tumors [10-12] which further paves the way for metastasis [13, 14].

Stress-induced angiogenesis and metastasis seem to play a balancing role to favor tumor growth and dissemination. The relationship between angiogenesis and 
metastasis appears to be in sync, enabling the anti-angiogenic therapies to reduce the metastatic propensity [14, 15]. Nonetheless, reports of metastasis stimulated by the anti-angiogenic treatment further demonstrate the complicated nature of this relationship [16, 17]. In stark contrast to the underlying complexities between these phenomena, currently available in vitro model systems seem far too simplistic to mimic the actual situation.

Traditionally, 2D monolayer systems are widely used to study metabolic stress conditions without any similarity with tumors. For example, experimental deprivation of glucose was found to induce cell death in vitro, while in xenograft studies, it has been found to activate angiogenesis through AMPK and ATF4 pathways [10,18]. Similarly, starvation of protein or amino acid (such as arginine) induces apoptosis in monolayer culture but not in the tumor, which evades death through the GCN2/ATF4 pathways [11, 19]. Scarcity of another micro-nutrient, oxygen, causes hypoxic stress in cell culture, followed by apoptosis and autophagy [20-23], but not in tumors $[12,13]$. These clearly show the discrepancy between the current functioning models and reality, which needs to be in sync to better understand this process.

Additionally, most current studies use a single stress condition in the experimental systems to simplify the outcome. In contrast, in a real dynamic scenario, the tumor is never exposed to any singular stress but multiple stresses which exert a collective effect. Despite this critical limitation, only one study has recognized the implications of multi-stress conditions so far [24].

The effect of nutrient stress on tumor metastasis or epithelial to mesenchymal transition (EMT) was studied in the past [25-27]. Moreover, the direct impact of metabolic stress has been studied primarily in two-dimensional (2D) platforms, which can not depict the complex nature of 3D tumors in terms of evasion or tolerance to stresses [28, 29]. The use of xenograft models in this scenario remains limited due to technical and analytical challenges. Furthermore, controlling experimental variables and envisioning the concept within the in vivo model system seem to be problematic [30] and expensive. In contrast, in vitro models such as multicellular spheroids, scaffolds, microfluidics, and others could provide a middle ground, offering more control over the variable parameters for studying such complex scenarios in a cost-effective system [31]. A recent study validates the similarities of the spatial distribution of metabolites between spheroids and cancer tissues, which makes it a prime model for metabolical studies [32].

The current study investigates the effect of multiple stress conditions on invasive and non-invasive breast cancer spheroids to study their impact on various behaviors, including tumorigenesis, spheroid formation, growth, phenotype, migration, and tumor-stromal crosstalk. It also aims to establish the multicellular spheroid as a cost-effective experimental platform to study tumor biology in vitro and validate its efficacy as a functional tumor model.

\section{Methods and materials}

\section{Materials}

DMEM with high glucose $(4.5 \mathrm{gm} / \mathrm{lt})$ and low glucose (1 $\mathrm{gm} / \mathrm{lt}$ ), fetal bovine serum, penicillin-streptomycin, TrypsinEDTA solution, and $\mathrm{CoCl}_{2}$ were procured from Himedia Laboratories, India. Trizol, Cytotracker red, Cytotracker green, and RevertAid first-strand cDNA synthesis kits were obtained from Thermo Fisher Scientific. TRITC conjugated phalloidin, DAPI, Rat Tail Collagen Type I, and Cultrex ${ }^{\mathrm{TM}}$ spheroid invasion matrix was brought from Sigma Aldrich, Merck, Germany. Oligonucleotides and GoTaq ${ }^{\circledR}$ Green PCR master mix are procured from Eurofin, India, and Promega. All other regular chemicals used were of cell culture grade and procured from Merck and Himedia, India. All everyday plastic wares were of tissue culture grade and brought from Biostar, Tarsons, and Nest.

\section{Cell culture}

MCF7 and MDA MB 231 cell lines were procured from NCCS, Pune, India, and maintained in DMEM supplemented with $10 \%$ fetal bovine serum and $1 \%$ penicillinstreptomycin solution. Human mesenchymal stem cells were isolated from human gingiva tissue described elsewhere [33], and cells from passages 10-11 were used. Cells have grown in humidified $\mathrm{CO}_{2}$ incubator at $37^{\circ} \mathrm{C}$ for 2-3 days to reach confluency followed by Trypsin-EDTA $(0.25 \%)$ solution-mediated harvest.

\section{Creating nutritional, hypoxia, and combined stress}

Different combinations of glucose \{ high ( $4.5 \mathrm{gm} / \mathrm{lt}$ glucose $)$ and low (1 gm/lt glucose) $\}$ and serum $(10 \%, 5 \%$, and $0 \%)$ are used as referred earlier [18, 24, 34]. Individual conditions such as control $(4.5 \mathrm{gm} / \mathrm{lt}$ glucose $+10 \%$ serum $), \mathrm{H}-5$ ( $4.5 \mathrm{gm} / \mathrm{lt}$ glucose $+5 \%$ serum $), \mathrm{H}-0$ ( $4.5 \mathrm{gm} / \mathrm{lt}$ glucose $+0 \%$ serum), L-5 ( $1 \mathrm{gm} / \mathrm{lt}$ glucose $+5 \%$ serum $)$, and L-0 ( $1 \mathrm{gm} / \mathrm{lt}$ glucose $+0 \%$ serum) were used to create macro-nutritional stress conditions. Breast cancer cells were cultured in 2D conditions treated with $\mathrm{CoCl}_{2}(50,100,150$, and $200 \mu \mathrm{M})$ to create pharmacological hypoxia (Hyp) and confirmed by expression of the hypoxia-related marker gene (Hif1 $\alpha$ ). Throughout the text, the combined stress conditions were mentioned as H-5-Hyp, H-0-Hyp, L-5-Hyp, and L-0-Hyp. 


\section{Effect of nutritional stress on in vitro tumorigenicity}

Clonogenic assay or soft agar colony formation assay was used to analyze the effect of individual and combined stress on the anchorage-independent proliferation of cancer cells as referred elsewhere [35]. Briefly, $1 \%$ melted agarose and stress condition media were equilibrated at $42^{\circ} \mathrm{C}$ and mixed in equal proportions to make a final concentration of $0.5 \%$ agarose. Next, the agarose solution $(250 \mu \mathrm{l})$ was layered on the 24-well plate and was allowed to solidify. Furthermore, $1 \%$ agarose was diluted with cells ( 2500 cells/well) to make a final concentration of $0.3 \%$, and $250 \mu \mathrm{l}$ of this solution was used to make the top soft agar layer. After solidification, 250 $\mu \mathrm{l}$ of stress media was added to the top layer. Medium was replenished periodically every 2-3 days, and the culture was maintained for 10 days to form visible colonies.

\section{Spheroid fabrication}

Multicellular spheroids of MCF7 cells were formed under normal conditions following the protocol as stated earlier [36]. Briefly, 24-well plate was coated with $1 \%$ agarose and allowed to solidify. Cellular suspensions (5000 cells/ well) were added to the agarose-coated wells. The plate was centrifuged at $2000 \mathrm{rpm}$ for $3 \mathrm{~min}$ and incubated for 3-5 days under standard cell culture conditions. Spheroids of 150-200- $\mu \mathrm{m}$ diameter were collected afterward for further processing.

Fabrication of MDA MB 231 spheroids was done by using three methods in this project. Firstly, the MDA MB 231 spheroids were fabricated using the low adhesion protocol as stated before for spheroid formation assay [36]. Secondly, Cultrex ${ }^{\mathrm{TM}}$ BME spheroid formation matrix was used to seed 3000 cells followed by 3 days of incubation and utilized for spheroid formation assay. Thirdly, MDA MB 231 spheroids were fabricated to analyze the effect of stress on growth, phenotype, and sub-cellular structures along with stem cell recruitment assay following a protocol described elsewhere [37] with some modifications. Briefly, the 96-well $\mathrm{U}$ bottom plates were coated with $1 \%$ agarose followed by the seeding of 5000 cells/well and centrifugation at 2500 rpm for $6 \mathrm{~min}$. After $24 \mathrm{~h}$, collagen $(3 \mu \mathrm{g} / \mathrm{ml})$ was added to the wells, followed by centrifugation at $500 \mathrm{~g}$ for $20 \mathrm{~min}$. Finally, the plate was incubated for 5 days to obtain the spheroids.

\section{Effect of nutritional stress on spheroid formation}

Nutritional and hypoxic stresses were induced individually and in combined conditions to create multiplex stress conditions. The effects of these stress conditions on spheroid formation were studied using the stress media to produce spheroids. Monolayer cells were harvested and subjected to spheroid formation protocol for $72 \mathrm{~h}$ under stress. After $72 \mathrm{~h}$, the formation of spheroids was confirmed, and the viability of those aggregates was further evaluated using Calcein AM $(1 \mu \mathrm{M})$ staining. The aggregation of the cancer cells under combined stress conditions was tested using the Cultrex ${ }^{\mathrm{TM}}$ spheroid invasion matrix following the manufacturer's protocol. Briefly, 3000 cells were seeded in the spheroid formation matrix and centrifuged for $200 g$ for $3 \mathrm{~min}$. The matrix was further incubated under standard cell culture protocol for $72 \mathrm{~h}$, followed by microscopic confirmation of spheroid formation.

\section{Analyzing the effect of stress on spheroid growth and phenotype}

The effect of individual and combined stress conditions on already formed spheroids was investigated by exposing the spheroids to the stress conditions. MCF7 and MDA MB 231 cells were harvested and seeded for spheroid formation as described above. Afterward, fabricated spheroids were collected and subjected to a range of individual or combined stress for $72 \mathrm{~h}$. Stressed spheroids were further used for subsequent analysis of morphology and size. Spheroid growth was analyzed by measuring the diameter using NIS software, and the values were plotted using Origin Lab software.

\section{Analyzing the effect of stress on the sub-cellular entity of breast cancer spheroids}

The effect of stress on cellular sub-structures within the MCF7 and MDA MB 231 spheroids was investigated by analyzing the nucleus and actin cytoskeleton appearance. Spheroids treated with individual stress were only used for this experiment as combined stress renders them very fragile. The control and stressed spheroids were washed with $1 \mathrm{X}$ PBS and fixed with 2-4\% PFA, followed by the permeabilization with $0.01 \%$ Triton X100 for 15 min. Nucleus staining with DAPI $(1 \mathrm{mg} / \mathrm{ml})$ was done for $15 \mathrm{~min}$ at room temperature. Nucleus imaging was done with $\times 60$ objective of CLSM (Nikon AR1), and the images were processed with ImageJ software. Measurement of the nucleus area of MCF7 spheroids was done by ImageJ software, and the values were plotted using a box-whisker plot.

The distribution of the actin cytoskeleton in control and stressed spheroids was analyzed by staining the actin filaments. The control and stressed spheroids were washed with 1x PBS and fixed with 2-4\% PFA, followed by the permeabilization with $0.01 \%$ Triton X100. The spheroids were further stained with TRITC conjugated phalloidin (1:100 dilutions) for $2 \mathrm{~h}$, followed by the imaging protocol as before. The thickness of cortical actin of MCF7 spheroids was measured by ImageJ as described elsewhere [38]. Briefly, the fluorescence intensity of the 8-bit image was measured and fitted 
using the Gaussian function in the Origin lab. FWHM of the fitted peak was used as a measure of thickness. A minimum ten cells per condition and a minimum of $5 \mathrm{ROI} / \mathrm{cells}$ were used for the measurement.

\section{Analyzing the effect of stress on mRNA level}

The effect of the individual and combined stress conditions on a few specific mRNA clusters' transcription levels was investigated further. MFC7 spheroids $(n>250)$ were harvested and exposed to different individual and combined stress conditions for $72 \mathrm{~h}$. Stressed and control spheroids were collected and used for RNA extraction. Briefly, the control and stressed spheroids were treated with Trizol-chloroform to isolate total RNA. Isolated RNA $(1 \mu \mathrm{g})$ was further used to prepare cDNA following the manufacturer's protocol (RevertAid first-strand cDNA synthesis kit). Finally, an equal volume of cDNA $(1 \mu \mathrm{l})$ was used to assess the mRNA clusters (detail in SI table 1) through semi-quantitative RTPCR using the GoTaq ${ }^{\circledR}$ Green Master Mix. PCR product was analyzed through agarose gel electrophoresis and quantified using the gel analysis plugin of ImageJ. $\beta-2$ microglobulin was used as an internal control throughout the study. The fold change values of each mRNA were calculated by first normalizing the mRNA expression level using their internal control followed by normalization using respective mRNA values of control. Normalized values above and below 1.0 were considered up- and downregulated. The fold change values were plotted using the Origin Pro software with standard error bars.

\section{Analyzing the relationship between stress and migration modality}

The migration ability of the stressed spheroids was studied through two different assays. MCF7 spheroids were used for both pseudo-3D (spheroid melting) and sprouting migration assay. On the other hand, MDA MB 231 spheroids were tested for sprouting assay.

\section{Pseudo-3D migration}

Already grown MCF7 spheroids were treated with individual and combined stress conditions for $72 \mathrm{~h}$. After the treatment, they were subjected to migration under two conditions: stress and rescue (fresh culture medium). Briefly, the spheroids were allowed to migrate on cell culture treated glass coverslips/24-well plates for 20-24 h in the presence of either stress or rescue medium. Phase-contrast images of migrating cells were acquired and analyzed. As collective migration is typical for epithelial cells, the conditions initiating mixed and single-cell migration conditions were investigated further. The capacity to induce single-cell migration by each type of stress condition (L-0, H-5-Hyp, and L-5-Hyp) was analyzed by measuring the invasion index. The number of single cells moving out of each spheroid was normalized with the initial spheroid area. The data were plotted using the violin plot of Origin Pro software.

Furthermore, MMP secretion assay and inhibitor assay were done for these three conditions. Briefly, 100s of stressed spheroids (L-0, H-5-Hyp, and L-5-Hyp) were treated with rescue media for $72 \mathrm{~h}$ and harvested later. Finally, the spheroids were lysed using RIPA lysis buffer, and the supernatant was used for a gelatine-based zymogram [36].

The modality of singly migrating cells was further established by differential inhibitor assay. The spheroids were allowed to migrate for $20-24 \mathrm{~h}$ in the presence of marimastat $(10 \mu \mathrm{M})$ (MMP inhibitor), ML7 $(10 \mu \mathrm{M})$ (myosin light chain kinase inhibitor), bisindolylmaleimide $\mathrm{I},(1 \mu \mathrm{M})$ (protein kinase $\mathrm{C}$ inhibitor), and blebbistatin $(10 \mu \mathrm{M})$ (myosin II inhibitor). The numbers of migrating cells in control and tested samples were counted using phase-contrast microscopy and plotted using Origin Pro in a box-whisker plot.

\section{Spheroid sprouting assay}

Both MCF7 and MDA MB 231 spheroids grown in Cultrex ${ }^{\mathrm{TM}}$ Spheroid formation and invasion matrix under combined stress conditions were utilized for sprouting assay. Briefly, stressed spheroids fabricated in the Cultrex ${ }^{\mathrm{TM}}$ Spheroid formation matrix were subjected to the addition of the invasion matrix along with the mouse laminin $(1 \mu \mathrm{M})$ and rescue media. The formation of sprout-like structures was monitored, followed by image acquisition after $72 \mathrm{~h}$.

\section{Effect of stressed spheroids on stromal cell migration and recruitment}

A cellular crosstalk assay was designed to investigate the effect of the stressed spheroid on the surrounding stromal population. First, both invasive and non-invasive spheroids were treated with combined stress conditions (H-5Hyp, H-0-Hyp, L-5-Hyp, and L-0-Hyp) for $72 \mathrm{~h}$. Next, the stressed spheroids were incubated with Cytotracker green ${ }^{\mathrm{TM}}$ for $30 \mathrm{~min}$ and transferred to agarose-coated 96-well plates, followed by the addition of $50 \mu \mathrm{l}$ collagen solution $(1 \mathrm{mg} /$ $\mathrm{ml}$ ). After the $\mathrm{NaOH}$-mediated collagen polymerization, pre-stained (Cytotracker red ${ }^{\mathrm{TM}}$ ) human mesenchymal stem cells were seeded $(10,000$ cells/well $)$ on top of the collagen layer supplemented with complete media. Migration and recruitment of MSCs over the next 24-72 h were monitored through CLSM. 


\section{Statistical analyses}

All experiments were repeated more than three times with a minimum of three technical replicates. For a normally distributed dataset, the significance of the difference between the mean value of the population was analyzed by parametric tests such as one-way ANOVA followed by post hoc testing such as Tukey's test (Origin Lab). The significance level used in all tests was 0.05 , and $p<0.05$ was considered significant.

\section{Results}

\section{Formulation of different levels of macro- and micro-nutrient stress}

Deprivation of individual macro-nutrient molecules (such as glucose and serum) and in combination with micro-nutrient (such as oxygen) (SI Fig. 1A) is assumed to create nutritional stress. By combining different stress conditions individually or together, five different stress levels, such as low, mid, high, severe, and extreme, are created for this study (Fig. 1A). Throughout the study, non-invasive (MCF7) and invasive (MDA MB 231) cell lines were used.

\section{Stress conditions influence the in vitro tumorigenesis}

The effect of the individual and combined stress on tumorigenesis is confirmed using a soft agar colony formation assay. For both cell lines, visible colonies were observed under control conditions, and the individual stress conditions did not significantly hamper the colony formation capacity. But the clonogenic capacity decreased as the stress level increased under combined conditions (SI Fig. 2).

\section{Stress conditions influence spheroid formation}

The spheroid-forming capacity of MCF7 and MDA MB 231 under stress conditions was studied further. As observed, individual stress conditions do not inhibit MCF7 cells from producing typical spherical aggregates with regular morphology. They maintain a compact structure with welldefined smooth boundaries. However, the increasing stress seems to impact the spheroid size negatively. The combined starvation of macro- and micro-nutrients affected spheroid formation efficiency significantly. As observed, the MCF7 spheroids lost their morphology and resembled the typical invasive spheroids (MDA MB 231) with irregular shapes. In this context, the invasive (MDA MB 231) cells perform way worse and barely form recognizable clumps under individual

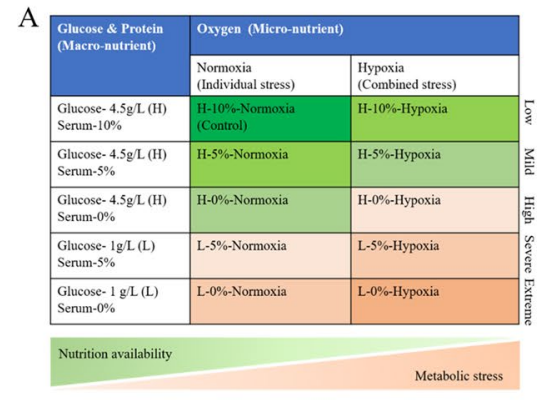

$\mathrm{C}$

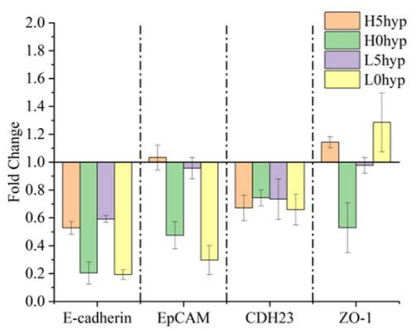

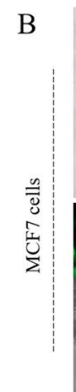
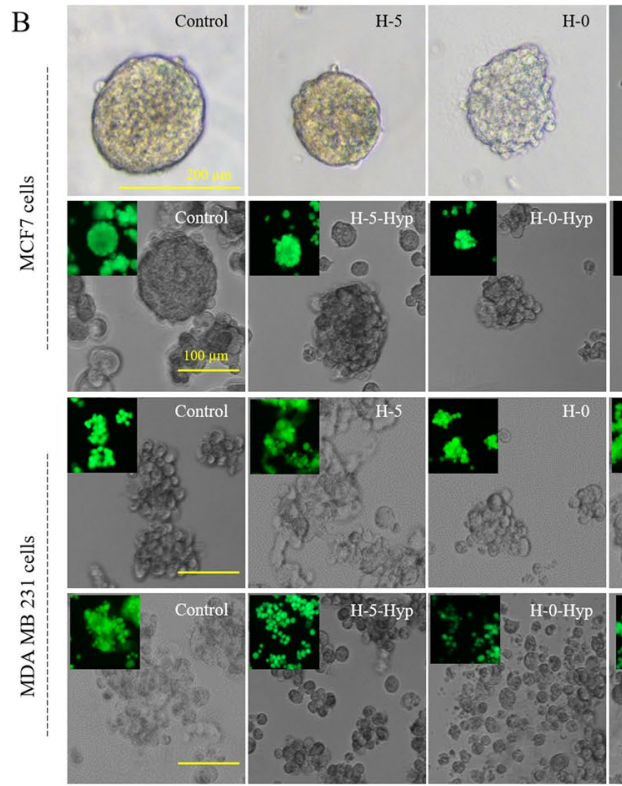
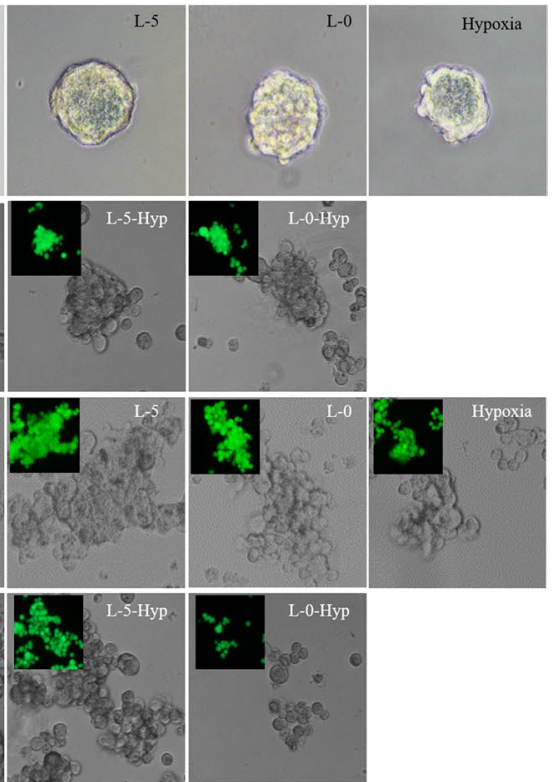

Fig. 1 Effect of individual and combined nutrient stress on spheroid formation capacity of non-invasive and invasive breast cancer cells. A A nutritional stress matrix is created by mixing different media components. A range of stress starting from low to the extreme is designed to understand the effect of different stress conditions on spheroid biology and behavior. B Non-invasive (MCF7) and invasive (MDA MB 231) cells were subjected to spheroid formation under individual and combined stress conditions under low-adhesion conditions. The inset images depict cell viability through calcein AM staining (green). C mRNA profiling of cell adhesion molecules (e.g., E-cad, EpCAM, CDH 23, and Zo1) was done from the MCF7 spheroid fabricated under individual stress conditions. Fold change values were plotted using the stacked plot of Origin Pro software. 
stress conditions. When subjected to combined stress, they did not even develop any aggregates. The viability of all these non-performer cells was confirmed by Calcein AM staining (inset) (Fig. 1B).

The ability to form spheroids or cellular aggregates was generally assigned to the availability and function of cell adhesion molecules (CAMs) such as E cadherin, EP-Cam, Zho 1, and Cad23, which form homo-dimers between cells and act as a nucleation point for aggregates. Minimal expression of cell adhesion proteins in invasive MDA MB 231 cells can be attributed to the formation of loose aggregates, as reported in multiple studies [39, 40]. However, MCF7 cells express a high quantity of CAMs, which is instrumental for compact spheroids formation [39]. Here, stressed MCF7 cells exhibit a few CAMs (E-cad and EP-Cam) downregulation, negatively influencing spheroid formation (Fig. 1C and SI Fig 1B). Minimal expression of CAMs in MDA MB 231 might become more impaired and inhibit cluster formation. Thus, we can conclude that the combined stress conditions can modulate the spheroid-forming capacity of MCF7 cells by manipulating the expression of CAMs.

As spheroid formation capacity in an ECM-less environment becomes severely weakened under combined stress, we next tried to find their influence in ECM-rich surroundings mimicking the in vivo situation. As we observed, spheroid formation happens within the Cultrex ${ }^{\mathrm{TM}}$ matrix for stressed MCF7 cells even under the combined stress and to a certain extent for stressed MDA MB 231 (Fig. 2A). Spheroids display compact structures with clear boundaries (H-5-Hyp, L-5, Hyp) but frayed edges under increased stress (H-0-Hyp, L-0-Hyp). However, for stressed MDA MB 231 cells, irregular and loose aggregates appear even in these ECM-rich favorable circumstances. Furthermore, the compactness of the spheroid decreased further with increasing stress. Therefore, we assume that the ECM environment can remedy the negative effect of mild to extreme stress conditions on spheroid formation. Previous literature suggested that ECM can circumvent the requirement of CAMs through integrin-ECM-mediated nucleation, which further employs E-cad-mediated compaction [41, 42]. However, we observed that ECM and integrin-ECM interactions are insufficient for the cells without ample E-cad expression (MDA MB 231). It is also feasible that the lowered expression of integrin $\beta 1$ might minimize the effect of cell-ECM bonding. Therefore, we presume that though cell-ECM interaction plays a vital role in tumorigenesis, it might not be the primary decisive factor under metabolically challenging conditions.

$$
\text { A }
$$

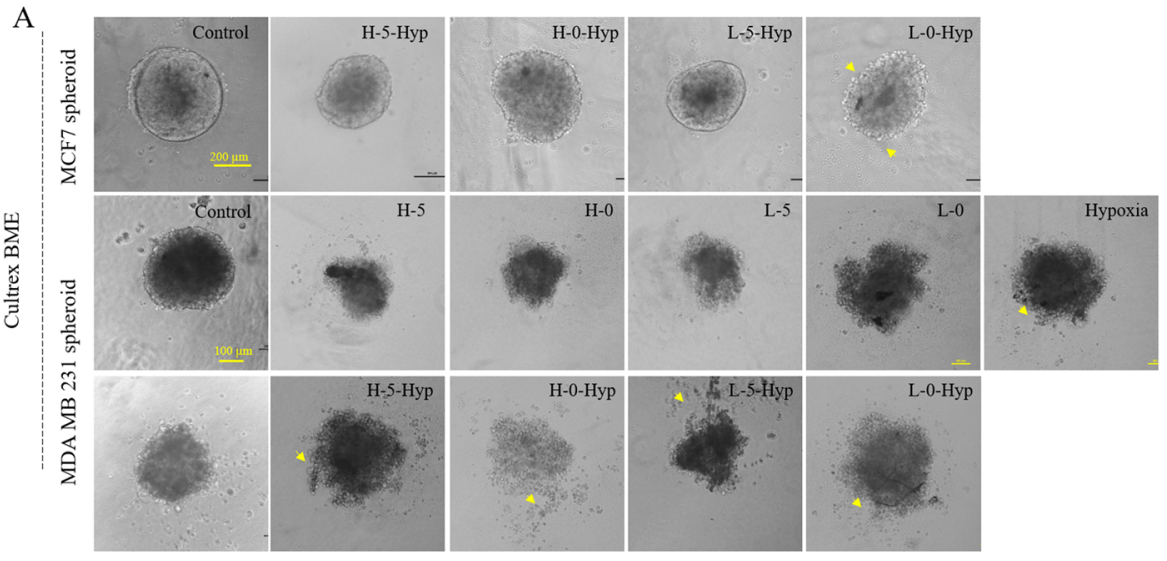

B

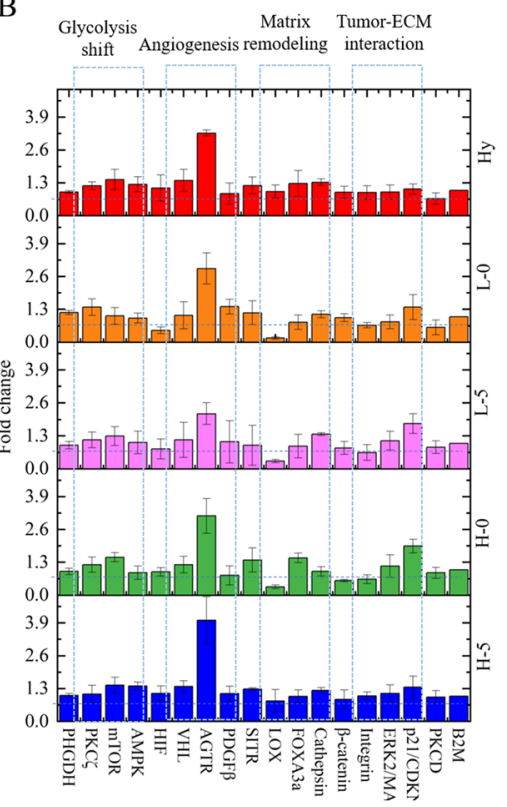

Fig. 2 Effect of individual and combined stress conditions on the formation of non-invasive and invasive spheroids within ECM-rich environment and mRNA profiling during spheroid formation. A Noninvasive (MCF7) and invasive (MDA MB 231) cancer cells were subjected to spheroid formation in a basement membrane extractrich environment. Compact spheroids of non-invasive cells and loose aggregates of invasive cells were observed after $72 \mathrm{~h}$ of incubation. B mRNA profiling of cancer hallmark genes (SI-Table 1) from the MCF7 spheroid fabricated under individual stress conditions. Fold change values were plotted using the stacked plot of Origin Pro software. The dotted boxes identify the differentially expressed gene clusters. The dotted black lines across the plots denote no fold change status (fold change value 1) compared to control values. Among the groups, angiogenesis (AFTR, PDGF, and SITR), matrix deformation (FOXA and cathepsin), and protein starvation (integrin $\beta 1$, ERK1/2, and P21) along with glycolysis shift (AMPK and mTOR) cluster showed upregulation. 
The formation of tumor-like structures or spheroids under stress conditions requires extensive manipulation of protein productions. mRNA profiling during spheroid formation was carried out to understand the effect of individual stress conditions on the transcription level of a few genes of interest (Fig. 2B). As expected, the mRNA of 17 gene candidates showed altered status compared to the control. A few of them (Hif-1 $\alpha$, FOXA3a, Lox, $\beta$-catenin, integrin $\beta 1$ ) exhibit downregulation with an increasing shortage of macro-nutrients, but not in hypoxic conditions. mRNA of the angiogenic cluster (VHL, AGTR, and PDGF $\beta$ ) exhibits significant upregulation in all stress conditions. Genes of ECM remodeling clusters (Lox, FOXA3a, and cathepsin) exhibit downregulation in macro-nutrient stress. However, the tumor-ECM interaction cluster (integrin, ERK, p21) remains stable in macro-nutrient stress conditions but altered in hypoxic conditions. Other candidates exhibit a similar profile among all the stresses. This data supports the hypothesis that spheroids can sense different stress conditions (macro or micro) and respond in diverse ways by manipulating their phenotype and behavior.

\section{Stress conditions influence the spheroid growth and morphology}

The previous experiments show that though individual (nutritional and hypoxic) stresses are not a deterrent to spheroid formation, combined stresses can inhibit the process entirely. Therefore, next, we tested the effect of stress conditions on already formed spheroids. The individual and combined stress conditions were observed to influence the spheroid morphology and size (Fig. 3).

MCF7 spheroids subjected to individual (nutritional and hypoxic) stress resemble the control sample morphologically with a well-defined periphery and compact nature. However, spheroid morphology happens to alter under combined stress conditions. For MDA MB 231, increasing individual stress seems to crumble the structure integrity, and combined stress conditions did this more severely (Fig. 3A).

Interestingly, high to extreme stress conditions such as H-0-Hyp and L-0-Hyp changed the spheroid morphology, exhibiting an irregular boundary similar to the spheroids prepared from invasive breast cancer cell lines (MDA MB 231). It is observed that among the individual nutritional stress conditions, the severe one (L-0) inhibits the growth significantly (Fig. 3B). The remaining stress conditions, along with hypoxia, exhibit no detrimental effect on spheroid growth. Remarkably, the high (L-5 and H-0-Hyp) stress seems to allow the spheroids to grow significantly compared to the control. Thus, it looks like a certain level of metabolic stress might activate tumor growth. Combined stress conditions mild (H-5-Hyp) and severe (L-5-Hyp) display no significant change in spheroid growth. Unexpectedly, in
A
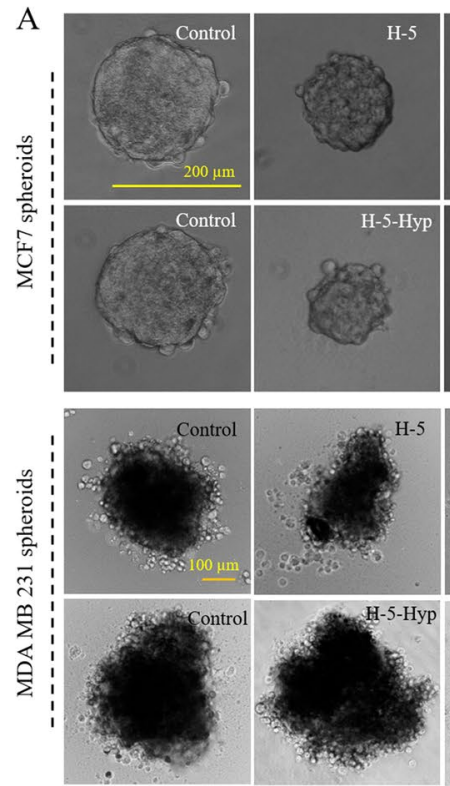
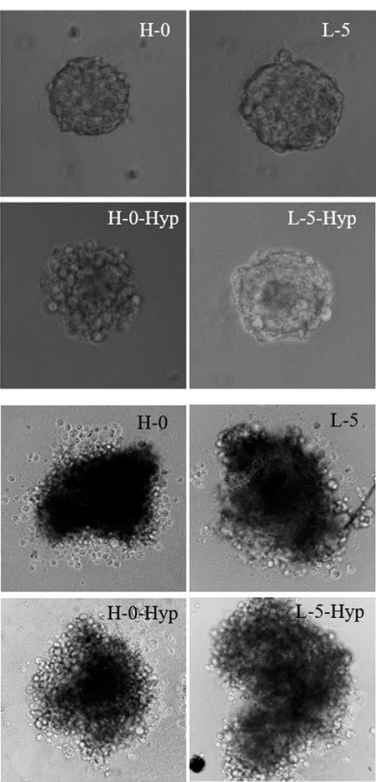
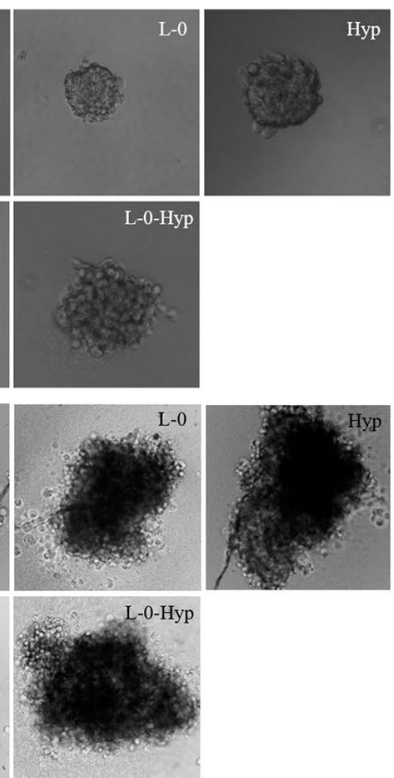

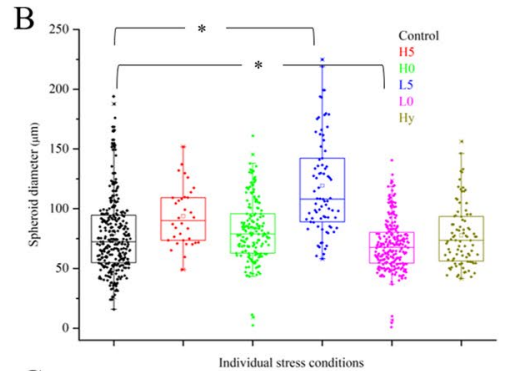

C

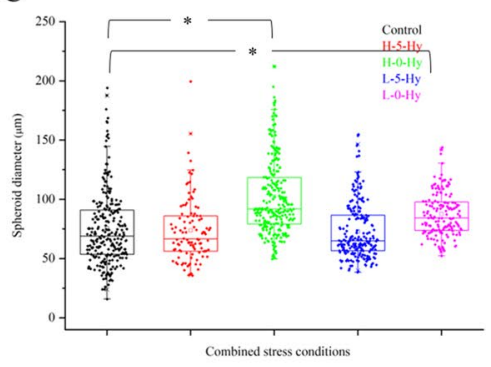

Fig. 3 Effect of individual and combined stress conditions on morphology and growth of already formed spheroids. A Already developed non-invasive (MCF7) and invasive (MDA MB 231) spheroids were exposed to individual and combined stress conditions for $72 \mathrm{~h}$. Combined stress (H-0-Hyp and L-0-Hyp) significantly changes the spheroid morphology of MCF7 spheroids, which now closely resembles the typical invasive spheroids (MDA MB 231). MDA MB 231 spheroids exhibit a disintegrated appearance with increasing stress. B Growth of MCF7 spheroids under stress conditions was measured by measuring the diameter after the exposure of $72 \mathrm{~h}$. Under individual stress, the spheroid sizes did not vary significantly from the control spheroids except L-5 and L-0 $(p<0.05)$. C Under combined stress conditions, both H-0-Hyp and L-0-Hyp displayed increased size compared to the control $(p<0.05)$ 
the high (H-0-Hyp) and extreme (L-0-Hyp) situations, the spheroid size remains equal to the control (Fig. 3C). This observation can be explained by the spheroid's loosened appearance, which increased the aspect ratio and influenced the size measurement.

From these results, it becomes clear that, once a tumorlike structure is created, the effect of metabolic stress has a limited impact on its growth. However, the metabolic stress might have a Gaussian proliferation rate, where an acceptable metabolically challenging environment might induce a positive outcome for the tumor (SI Fig. 3).

\section{Nutritional stress and its effect on the sub-cellular components of spheroids}

The effects of individual stress conditions on sub-cellular components such as nucleus and actin cytoskeleton were studied (Fig. 4). No significant alteration in the nucleus morphology of MCF7 and MDA MB 231 spheroids was observed (Fig. 4A). The nucleus area of MCF7 spheroids was measured, and $\mathrm{H}-0$ and $\mathrm{L}-0$ conditions showed a significantly smaller nucleus than control (Fig. 4B). It must be remembered here that the nucleus from only the top few layers of cells from a typical spheroid $(150 \mu \mathrm{m}$ diameter $)$ can be measured through CLSM. Despite being under metabolic stress, negligible apoptotic bodies are observed in low, high, and severe (Hypoxia, L-5, and L-0), highlighting the anti-apoptotic feature of the 3D structure. Additionally, serum deprivation caused the "cell-in cell" phenomenon, specifically in high-stress (L-5) conditions (SI Fig. 4). There was no difference observed in nucleus morphology in MDA MB 231 spheroids between control and stressed conditions, except a few apoptotic bodies are in hypoxic condition.

The distribution of the actin cytoskeleton was found to be similar among the control and test spheroids of both cell types (Fig. 4c). However, the actin cytoskeleton distribution in MDA MB 231 is different from that of MCF7 spheroids. Few cells with cortical actin were observed, but the typical distribution of actin cytoskeleton is network-like. The MDA MB 231 spheroids were composed of both elongated and circular cells. However, the canonical cortical actin ring, prevalent among all the cases, exhibits a change in its width when measured (Fig. 4D). Conditions like H-0 and hypoxia have shown the lowest thickness compared to control and probably play an essential role in maintaining their surface tension and other physical properties. Additionally, L-5, L-0,
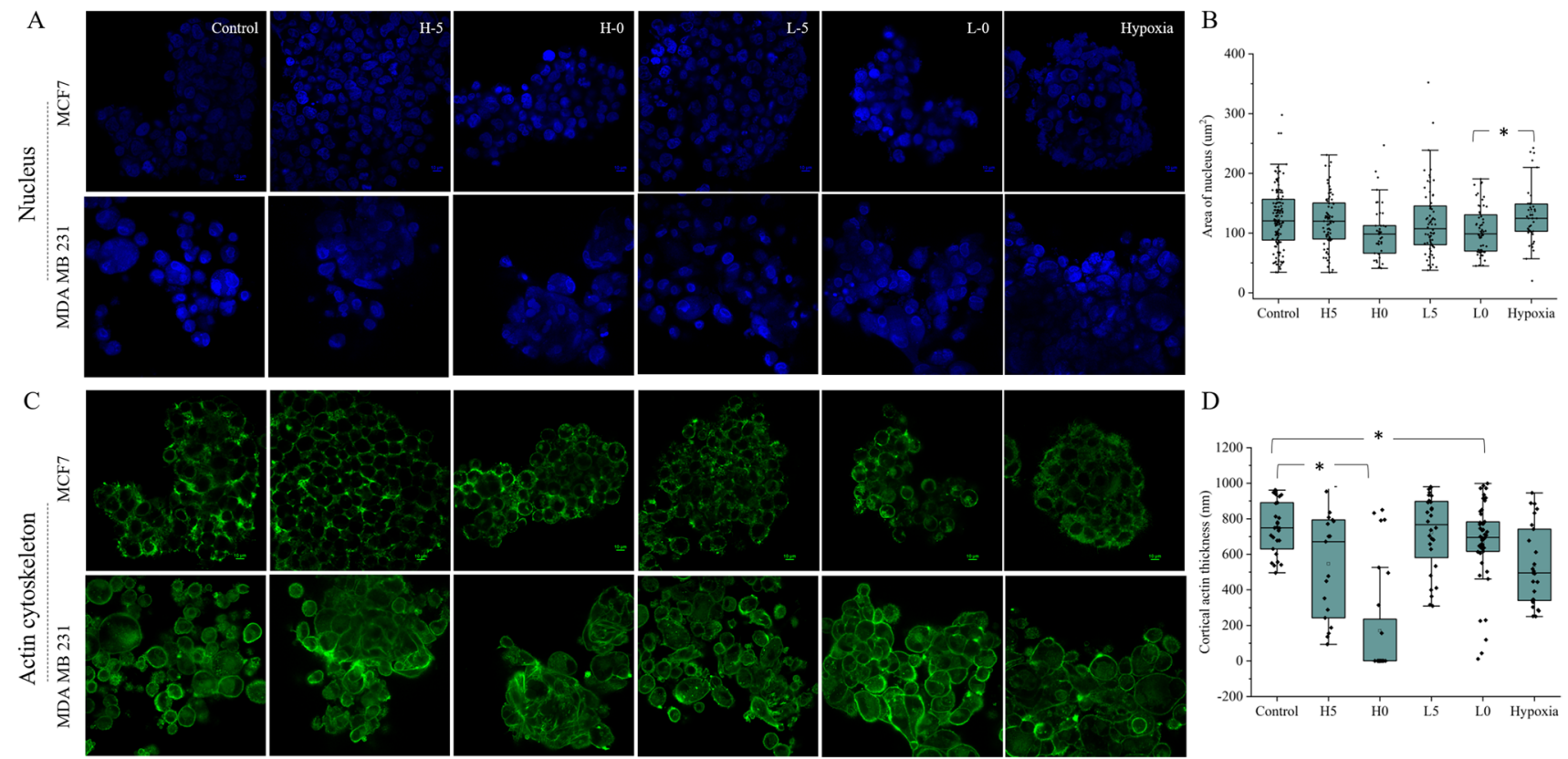

Fig. 4 Effect of nutrient stress on the sub-cellular entities of noninvasive and invasive spheroids. A Nucleus morphology of individually stressed non-invasive (MCF7) and invasive (MDA MB 231) spheroids was investigated after fixation and permeabilization followed by DAPI staining. B Nucleus size of spheroids (MCF7) was measured using the area measurement tool from ImageJ. Only the L-0 condition showed a significant decrease in nucleus size than hypoxic nuclei $(p<0.05)$. C Distributions of the actin cytoskeleton in stressed non-invasive (MCF7) and MDA MB 231 spheroids were studied under individual stress after fixing and permeabilization followed by staining with TRITC conjugated phalloidin. No significant difference in cortical actin ring was observed in MCF7 spheroids except the formation of actin-rich cytonemes in some cases (L-5, L-0, and hypoxia). On the contrary, MDA MB 231 spheroids exhibit actin stress fibers and stellate cells along with circular ones. No significant difference with control is observed. D Thickness of cortical actin of spheroids (MCF7) was measured fitted using Gaussian function in Origin Pro software. FWHM of the fitted peak was used to measure the thickness and plotted using the box and whisker plot 
and hypoxic conditions were observed to induce the occurrence of actin-rich membrane protrusions or cytonemes on the peripheral cells as compared to the control spheroids (SI Fig. 5).

\section{Nutritional stresses influence the mRNA transcription level}

The effect of individual and combined stress conditions on the transcription level of a few genes was analyzed (Fig. 5). Gene clusters belonging to different tumor activities such as glycolysis shift, angiogenesis, hypoxia, matrix deformation, and protein starvation were assessed. Low to severe stress conditions such as (L-5, L-0, and hypoxia) are observed to upregulate the mRNAs related to hypoxia (Hif-1 $\alpha$, VHL), glycolysis shift (mTOR, PKC $\zeta$ ), angiogenesis (AGTR, PDGF, SITR), protein starvation (P21, ERK 1/2), and matrix deformation (LOX, FOXA3a, Cathepsin). All individual stress significantly downregulated some genes, including integrin, $\beta$-catenin, PKCD, and PHDGH (Fig. 5A).

Combined stress conditions were seen to minimize the mRNA transcription level in general, except angiogenesis (AGTR, PDGF, SITR), ECM deformation (LOX, FOXA3a, Cathepsin), and protein starvation (P21, ERK 1/2) (Fig. 5B). Increased transcription of angiogenesis cluster highlighted the importance of multiplex stress conditions and inclusion of hypoxia. When compared between individual and combined panels, it becomes clear that combined stress creates a globally dormant state but intensifies specific clusters for a very directed way of function. These activated clusters highlight the altered metabolism and behavior of the spheroids under stress. Genes from glycolytic shift and protein starvation clusters might influence the spheroids to bypass the metabolic stress. Upregulated genes of angiogenic and matrix deformation clusters might initiate migration and neo-angiogenic growth. Minimal levels of cell-ECM adhesion-related genes (integrin $\beta 1$ and $\beta$ catenin) might influence the migration property of the spheroids, as observed in the following experiments.

\section{Stress and rescue influence the migration profile of spheroids}

The effect of stress conditions on spheroid migration is analyzed by in vitro pseudo-3D migration. In continuous stress (stressed spheroids subjected to migration under respective stress conditions), spheroids exhibit no migration and do not adhere to the surface (SI Fig. 6). The lack of adhesion can be explained by the lack of integrin $\beta 1$, as observed in previous mRNA profiling. In addition, lack of CAMs and ECM binding receptors might hamper focal adhesion formation and subsequent migration. On the other hand, rescued spheroids exhibit different migratory behaviors (SI-Table 2). As seen, the non-invasive spheroids (MCF7) typically pursue epithelial (collective) migration under control conditions, but others exhibit mixed/single cell or no migration (Fig. 6A). An exciting profile emerged, where the two extreme conditions (H-0-Hyp and L-0-Hyp) exhibit complete dormancy
Fig. 5 mRNA profile of few candidate genes transcribed in non-invasive spheroids exposed to individual and combined stress conditions. A Fabricated MCF7 spheroids were exposed to individual stress conditions for $72 \mathrm{~h}$. mRNA of glycolysis shift, hypoxia, angiogenesis, ECM deformation, and protein starvation clusters showed significant upregulation compared to control. In addition, transcription of all these 17 genes goes down in the $\mathrm{H}-0$ condition, giving it a transcriptomically inactive status. B Under combined stress, most of the genes' transcription level gets downregulated except the upregulation of angiogenesis, ECM deformation, and protein starvation clusters compared to control
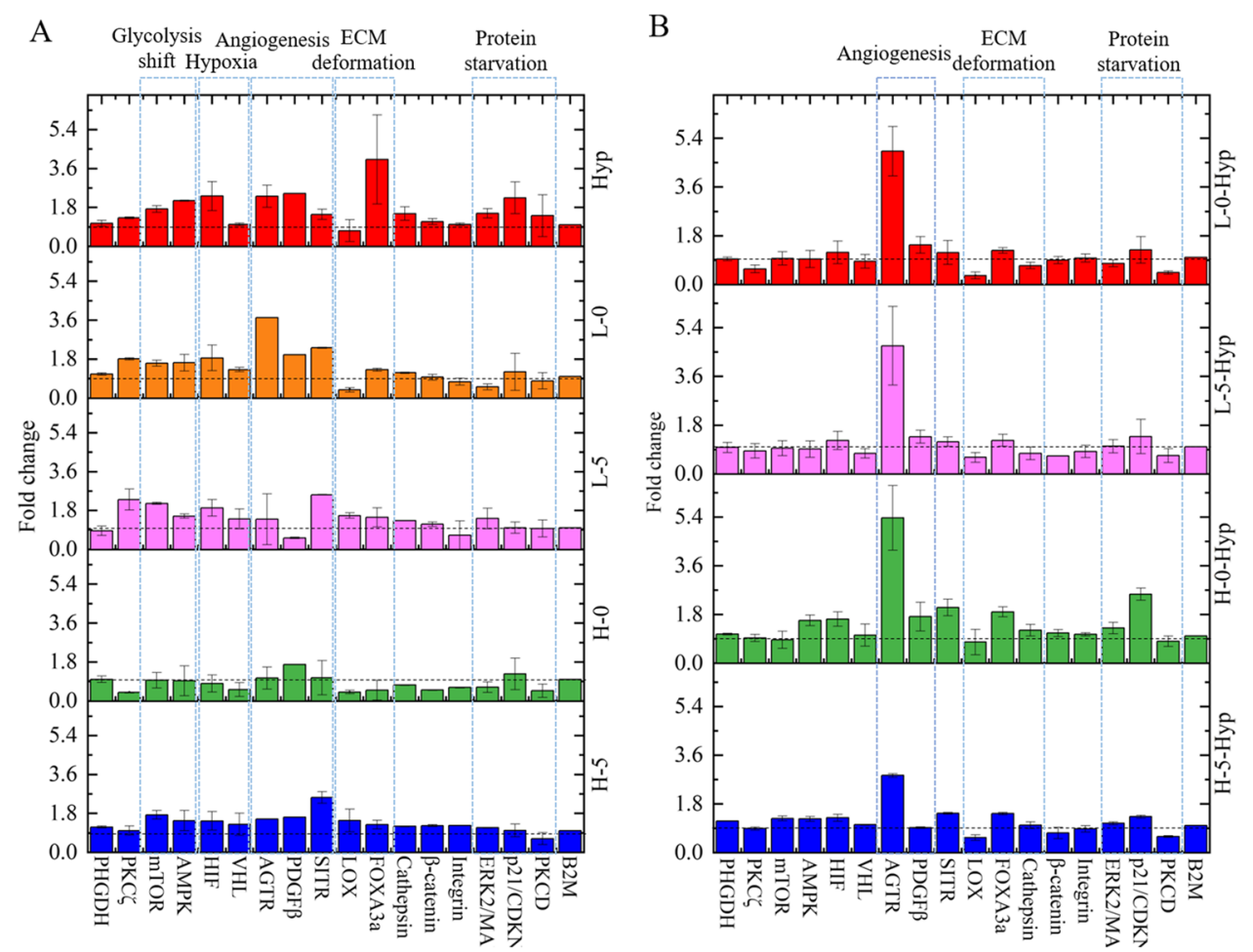
and severely stressed spheroids show only single-cell (L-0) or mixed mode of migration (H-5-Hyp and L-5-Hyp). Low and moderately stressed spheroids (H-5, H-0, L-5, hypoxia) show collective epithelial migration as typical control spheroids.

Further probing was done to understand the modality of single-cell migration in mild-severely stressed spheroids (L-0, H-5-Hyp, and L-5-Hyp). An invasion index analysis among these three conditions showed H-5-Hyp and L-5Hyp conditions as significantly invasive (Fig. 6B). Thus, we ascertain that hypoxia and macro-nutrient stress have a cumulative effect and modulate the migration profile to intensify the invasive quality. Furthermore, the zymogram of secretory MMPs by these spheroids (L-0, H-5-Hyp, and
L-5-Hyp) exhibits a minimal expression of MMPs (SI Fig. 7), increasing the likelihood of MMP-independent migration of these cells. Differential inhibitor test further supports this notion when marimastat, a broad-range MMP inhibitor, failed to inhibit the single-cell migration. Inhibitors such as blebbistatin, ML7, and bisindolylmaleimide I were used to identify the potential modality of singlecell migration. Only ML7 exhibits maximum inhibition (Fig. 6C and SI Fig. 8) and highlights the potential role of the myosin light chain kinase-mediated pathway (Fig. 7).

In chemo-attractant-mediated sprouting assay, the rescued spheroids exhibit increased sprout formation and single cells compared to control. Like MCF7 spheroids, rescued MDA MB 231 spheroids in combined stress conditions

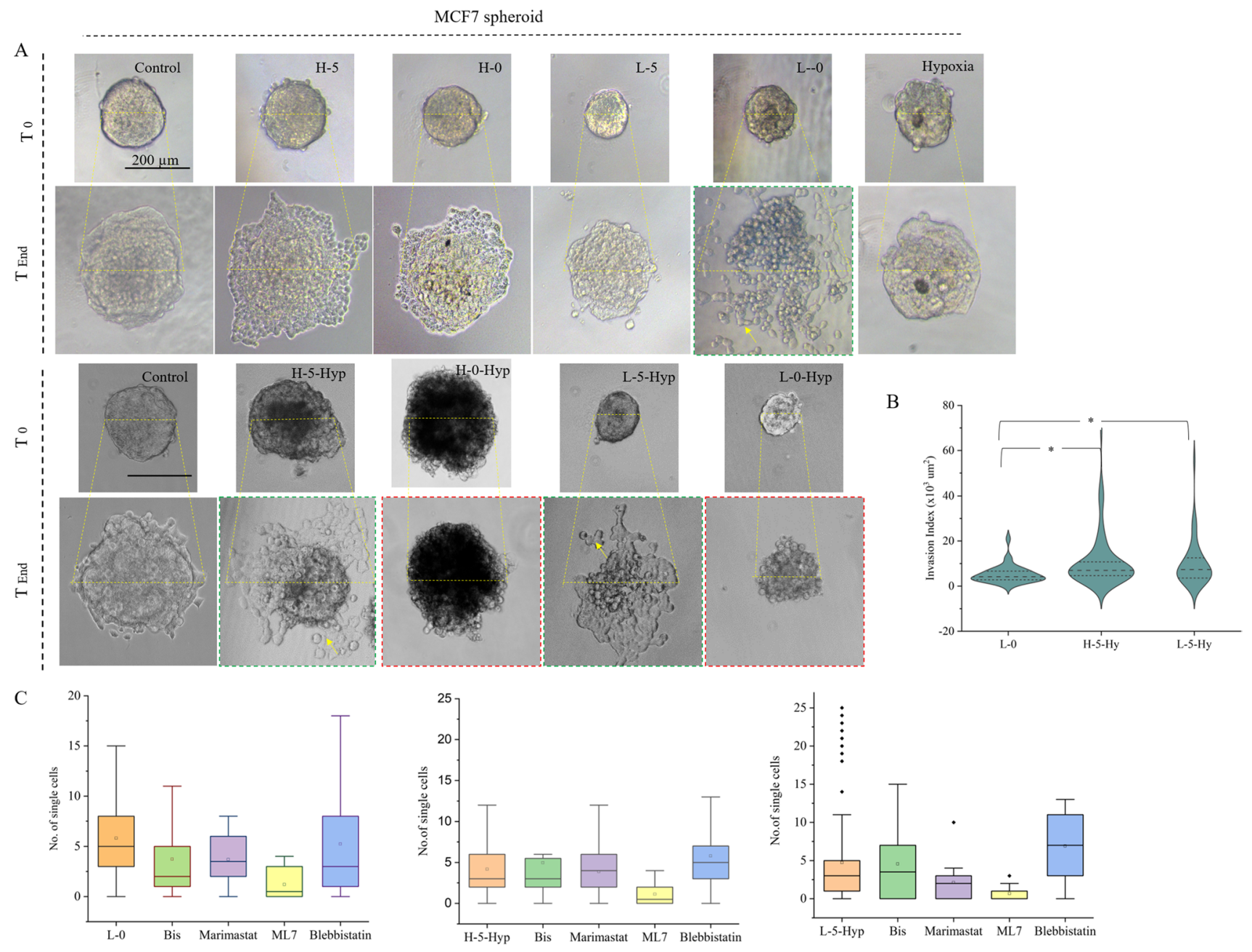

Fig. 6 Effect of individual and combined stress on non-invasive spheroid migration after rescue. A Fabricated MCF7 spheroids were subjected to individual and combined stress for $72 \mathrm{~h}$ followed by a rescue with fresh cell culture media and analyzed for adhesion and subsequence migration. Except H-0-Hyp and L-0-Hyp conditions (red border), all other spheroids adhered to the surface and migrated successfully. L-0, H-5-Hyp, and L-5-Hyp (green border) exhibit mixed/ hybrid migration. Yellow arrows denote the single cells. A yellow dotted line was used to compare spheroid size at the t0 and tend of the experiment. B Efficiency and modality of mixed migration mode under L-0, H-5-Hyp, and L-5-Hyp conditions were analyzed. Both H-5-Hyp and L-5-Hyp show higher index values compared to L-0. C Different inhibitors of single-cell migration, marimastat, ML7, bisindolylmaleimide I, and blebbistatin were used to inhibit the single-cell migration in L-0, H-5-Hyp, and L-5-Hyp conditions. 
(H-5-Hyp, H-0-Hyp, and L-5-Hyp) exhibit mixed modalities of migration, including cellular strands and single cells. However, the extreme stress condition (L-0-Hyp) showed no sprouts even after being rescued. Therefore, we assume that irrespective of their invasion status, the migration profile of both non-invasive and invasive spheroids is similar under the stress and rescue process. Additionally, pharmacological hypoxia emerges as a critical component, triggering the differential migration modality in invasive and non-invasive spheroids.

\section{Metabolically stressed spheroids influence stem cell migration and tumor infiltration after rescue}

The cellular crosstalk between the spheroid and stem cells was evaluated by the impact of stressed (H-5-Hyp, H-0-Hyp, L-5-Hyp, and L-0-Hyp) spheroids on the stromal population. The stressed spheroids (MCF7 and MDA MB 231) attracted the stem cells, and within $24 \mathrm{~h}$ of incubation, the stem cells were recruited into the spheroids (Fig. 8). The non-migratory nature of the stem cells without any inducer becomes apparent in no spheroid control. However, in the co-culture environment, the stem cells exhibit a downward migration towards the spheroids. In control spheroids, migrated stem cells surround the spheroid with minimal recruitment. With increasing stress, the recruitment of stem cells within the spheroids becomes more apparent as more red cells are visible within the spheroids. We concluded that the stressed spheroid exhibits more efficient recruitment, whereas the migration rate remains similar in all co-culture conditions.

\section{Discussion}

The cellular mass of a solid tumor increases with time and evolves into a metastatic one, presumably due to its inherent invasive nature [36]. However, during such growth, the availability of nutrients plays a critical role in reshaping the fate of that tumor. During the primary growth phase, tumor cells show unprecedented proliferation and excessive appetite. Such appetite often resulted in the incompetent utilization of available nutrients such as glucose through lactic acidosis $[8,9]$. In addition, growing tumors can also create oxygen deprivation due to the unavailability of blood capillaries, which increases nutrient deficiency further [29]. The influence of such metabolic stresses, including macro- (glucose and protein) and micro-nutrients (oxygen), on tumor growth and behavior is critical $[5,12,14]$.

Here, the stress conditions (minimal to extreme) are created by withdrawing macro- (glucose and protein) and

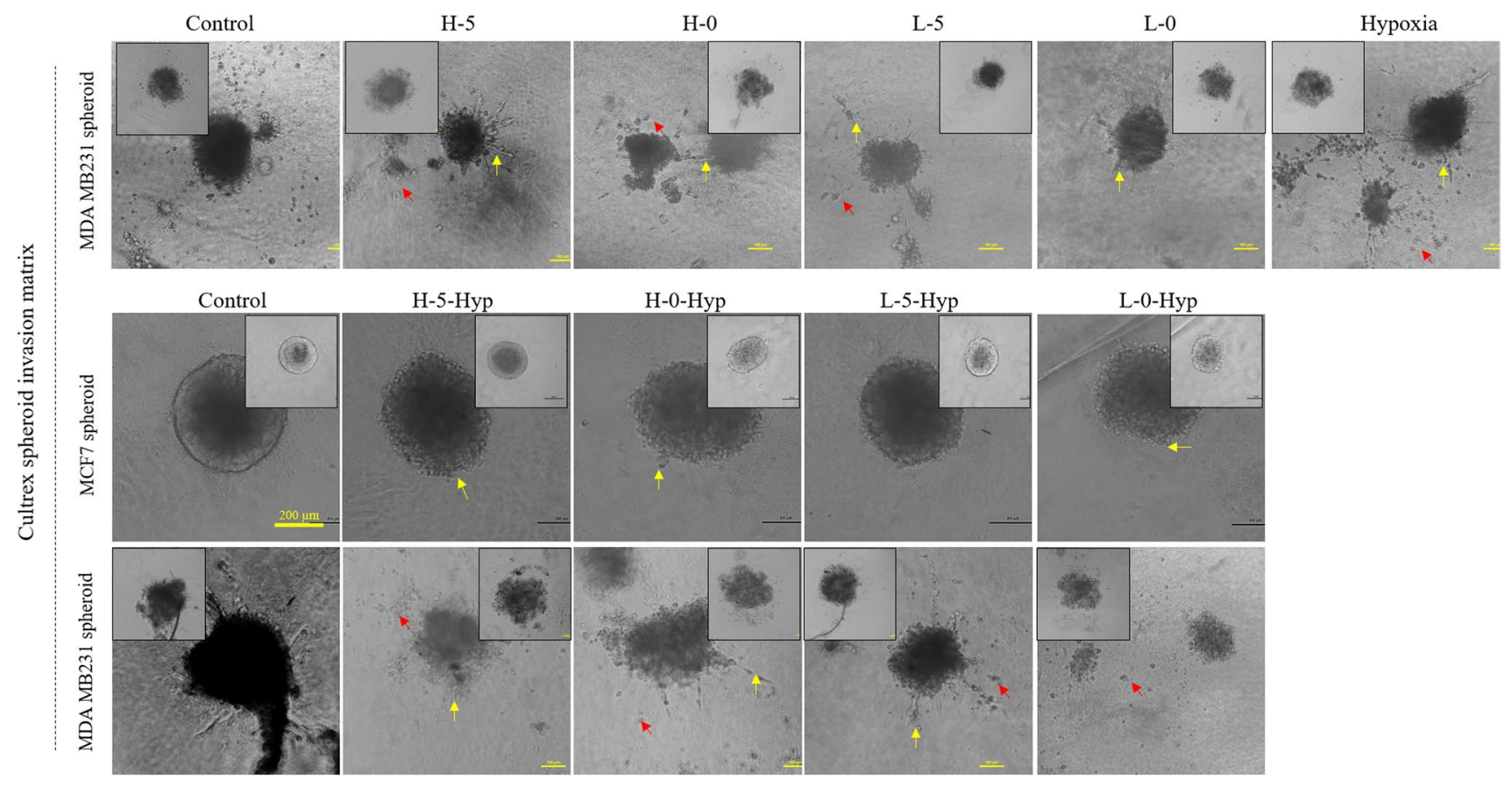

Fig. 7 Effect of stress and rescue on cellular migration of non-invasive and invasive within the extracellular matrix. Invasive (MDA MB 231) and non-invasive (MCF7) spheroids formed in the Cultrex BME spheroid formation matrix under individual and combined stress conditions subjected to chemotactic migration under the influence of laminin and rescue media. Sprout-like structures (yellow arrow) and migrating single cells (red arrows) were formed under all the stress conditions for invasive spheroids. Although non-invasive spheroids typically exhibit no migration in the ECM environment, here cellular strand formation (yellow arrow) was observed within $72 \mathrm{~h}$. Inset shows the spheroids at $t 0$. 
Fig. 8 Nutritionally stressed non-invasive and invasive spheroids influence stromal cell migration and recruitment. Prestained non-invasive (MCF7) and invasive (MDA MB 231) spheroids (green) were fabricated and exposed to combined stress conditions (H-5-Hy, $\mathrm{H}-0-\mathrm{Hy}, \mathrm{L}-5-\mathrm{Hy}$, and L-0-Hy) for $72 \mathrm{~h}$, followed by the rescue and interaction with mesenchymal stem cells (red). In no spheroid control, stem cells display no migration, while they tend to migrate towards the spheroids. In the left panel, the recruitment profile exhibits increased recruitment in stressed spheroids compared to control. The migration distance for stem cells within $24 \mathrm{~h}$ was approx. 1.6-1.7 mm

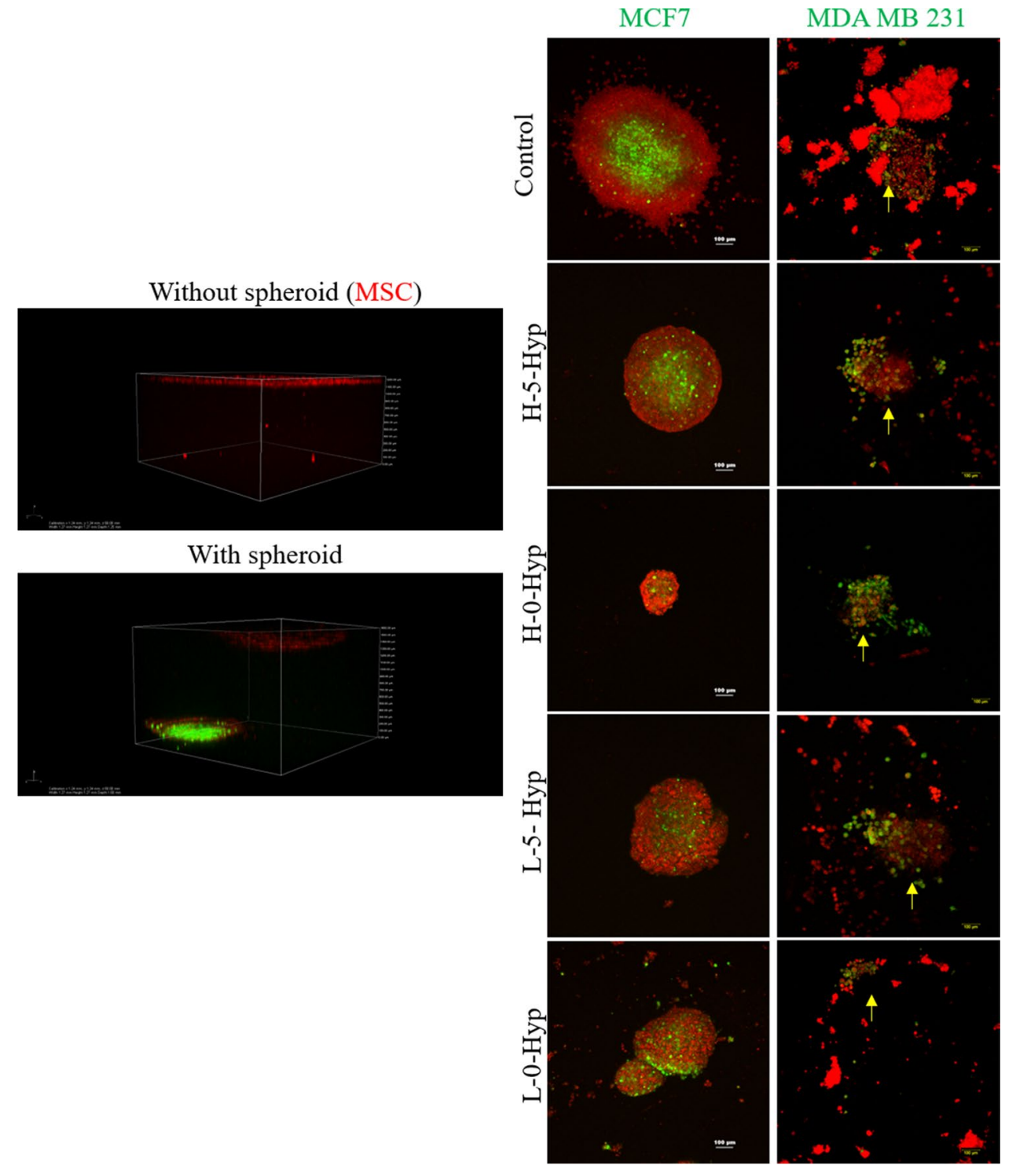

micro-nutrients (oxygen). Their effect on in vitro tumorigenesis and spheroid formation highlighted the importance of macro- and micro-nutrients during the process. The non-invasive cells seem to resist the individual stress conditions better and form colonies in soft agar assay. However, the invasive cells failed to generate the colonies under similar stress. Interestingly enough, combined stress conditions inhibit both the non-invasive and invasive cells from forming colonies. The in vitro tumorigenesis assay is in line with the spheroid formation efficiency [43, 44]. As observed, the spheroid formation ability of both invasive and non-invasive cells was significantly diminished under individual and combined stress conditions. Though individual stress conditions did not inhibit the formation of compact cellular aggregates in non-invasive cells, the combined stress conditions did so without compromising their viability.
Subsequently, we observed that ECM (or collagen) could circumvent the metabolic stress-mediated effect for the noninvasive cells and allow spheroid formation. The nucleation for tumorigenesis is a two-step process that includes integrins (cell-ECM contact) followed by the CAMs (cellcell contact) [41, 42]. Here, the inability of MCF7 spheroid formation without the ECM can be explained by the downregulated CAMs, which is in line with other reports [45]. However, the presence of ECM may promote the cellECM interactions via integrin $\beta 1$ while compensating for the decreased cadherin-mediated cell-cell adhesion [40]. Though the expression of integrin $\beta 1$ does not alter much in MCF7 cells under combined stress, its altered trafficking to the membrane might play a critical role [46]. On the other hand, the complete inability of stressed MDA MB 231 cells to form spheroid even with ECM can be explained by the absence of E-cadherin, N-cadherin, and Claudin 1 [47]. 
Within the ECM, the minimal expression of integrin $\beta 1$ does not seem enough to salvage the stressed MDA MB 231 cells. This observation highlighted the essential nature of cell-cell adhesion for tumorigenesis over cell-ECM interaction under a metabolically challenged environment. The reduction of homotypic cell adhesion molecules also explains the altered morphology of the formed spheroids from a well-defined compact structure to irregular-shaped loose aggregates. This observation is also in line with previously reported mathematical modeling, predicting the possible morphological alteration under metabolic stress $[48,49]$. The transformed appearance resembles the invasive spheroids created in vitro (MDA MB 231) [36] with minimal CAM expression [50, 51] and aggressive cancerous masses $[52,53]$. We propose that the exposure to nutritional stress during the tumorigenesis might re-program the non-invasive spheroid to the quasiinvasive one, exhibiting the phenotypical changes. During tumorigenesis or spheroid formation, cells are observed to re-program their transcriptomic profile, which includes the matrix deformation, protein starvation, angiogenesis, hypoxia, and glycolysis shift clusters. Upregulation of specific groups such as matrix deformation and angiogenesis insinuate the need to circumvent the metabolic stress.

Already formed spheroids behave differently under individual and combined stress conditions, further implying the importance of multiplex stress-related studies. Neither the individual nor the combined stress conditions negatively impacted the size of already formed spheroids. However, the loose assembly of MDA MB 231 aggregates disintegrates quickly under stress. As expected, only L-0 samples exhibit minimal growth compared to the control. Therefore, it can be assumed that nutrient deprivation does not have any detrimental effect on tumor growth after the formation of multicellular structures. Combined stress conditions on tumor growth show change in spheroid morphology under high and extreme stress (H-0-Hyp, and L-0-Hyp) mimicking the MDA MB 231 aggregate. The spheroids under these conditions exhibit a larger size compared to the control. The loosened-up appearance can explain the appearance of larger size under high and extreme stress. Such phenomenon is also observed in a recent study where cells under serum deprivation exhibit increased cell area [54]. Though no negative impact of stress conditions on spheroid growth is observed, an interesting observation emerges from both individual and combined conditions. The Gaussian distribution of the spheroid sizes exhibits increased size in L-5 and H-0-Hyp conditions, which might be significant as both belong to our study's "high" stress conditions. It might indicate the positive relationship between an endurable stress level and unfettered tumor growth through fatty acid metabolism [55]. Though the elimination of glucose and serum reportedly initiates cell death in the monolayer cultures [56, 57], it shows no adverse effect on spheroid growth.
The effect of stress on the transcriptomic profile of already grown spheroids exhibits a different profile. As observed, the H- 0 condition shows minimal transcriptomic activity for the candidate genes here, whereas H-5, L-5, and L-0 display similar profiles with upregulated mRNAs for PKC, mTOR, AMPK, SITR, LOX, and FOXA3a. Hypoxia, as individual stress, showed no visible impact on tumorigenesis or growth but altered the mRNA profile of $\mathrm{p} 21$, PKCD, MAPK, FOXA3a, HIF, AMPK, and mTOR cluster significantly as reported earlier $[18,20]$. Under the combined stress, mRNA of AGTR and PDGF $\beta$ remains undetectable under all the conditions. Unavailability of these mRNA might indicate the obstruction of endothelial cell recruitment and formation of new blood vessels [58]. Additionally, among these conditions, only $\mathrm{H}-\mathrm{O}-\mathrm{Hy}$ showed upregulation of p21, FOXA3a, HIF, AMPK, and SITR mRNA, probably due to the hypoxia.

Analysis of sub-cellular entities such as the nucleus under individual stress conditions reveals that deprivation of glucose and protein for $72 \mathrm{~h}$ is not enough to inhibit cell division and induce cell death which contradict the previous reports from the monolayer culture [59, 60]. The minimal occurrence of apoptotic cells can be explained by the anti-apoptotic nature of the three-dimensional structures of spheroids [61] and the availability of a TRAIL-resistant population [62]. Additional sporadic observations such as multi-nucleation and cell-in-cell phenomena can be explained by the mitotic stress [63] and entosis [64, 65]. No significant change in nucleus size is observed except $\mathrm{H}-0$ and L-0, which display smaller nuclei probably due to the nuclear recycling process, namely nucleophagy to tackle the nutrient-deprived conditions [66]. As the cellular shape and internal transport depend on cytoskeletal distribution, further analysis of actin cytoskeleton is carried out in stressed spheroids. The distribution of actin filaments as a cortical mesh in MCF7 spheroids displays no modification due to metabolical stress. However, decreased thickness of the cortical ring is observed in certain conditions ( $\mathrm{H}-0$ and hypoxia). On the other hand, the actin distribution in MDA MB 231 spheroids differs from that of MCF7 spheroids. The actin cytoskeleton in MDA MB 231 spheroids depicts both circular and stellate cells in the spheroid. Mostly, the stellate cells are present on the surface. This type of morphology in MDA MB 231 spheroids was reported earlier [67], supporting their invasive property. Stress may have bestowed more aggressiveness in the MDA MB 231 spheroids, which needs further probing. The effect of cortical actin on migration is unknown; however, in monolayer culture, decreased thickness of cortical actin is assumed to influence the surface tension [68] and cellular traction [38], which might imply a similar effect here. Additionally, certain stress conditions (L-0, H-0, and hypoxia) increased the membrane ruffling and formation of actin-rich macropinocytes or cytonemes. The 
abundance of such structures in stressed spheroids might influence the entosis or nutrient-scavenging mechanisms to survive in nutrient-deprived environments [69, 70].

Along with the tumorigenesis, spheroid morphology, growth, and sub-cellular organization, it is assumed that metabolic stress might also affect the migration modality of the tumor. Under continuous stress, spheroids exhibit no migration and maintain a quiescent stage, which is in line with the previous reports of tumor dormancy [71]. We assume that continuous stress might negatively influence integrin expression or its trafficking pathways [46], minimizing the capacity of cell-substratum adhesion [72] and subsequent migration. Further probing of such dormant tumors might help in understanding the survival mechanisms and endurance of environmental stresses. In addition, it might become instrumental in understanding the metastasis and re-colonization. Fasting has been selectively shown to inhibit the initiation and progression of both $\mathrm{B}$ cell and $\mathrm{T}$ cell lymphoblastic leukemia [73]. Therefore, tumor dormancy under continuous stress can be used as a model to understand the relationship between fasting and inhibition of tumor progression.

The awakening of the stressed spheroids through a nutritional rescue is critical. When rescued, diverse patterns of cellular migration emerged, which points out the quasi-invasive nature of the non-invasive spheroids (SI-Table 2) and highlights their migrational plasticity. Rescued spheroids exhibit a spectrum of migration modalities ranging from the collective (low-mild-high stress), single-cell (severe), mixed (mild-severe stress), and no (high-extreme stress) migration. When rescued, spheroids under high (H-0-Hyp) and extreme stress (L-0-Hyp) display dormant behavior. This may be due to the fact that serum deprivation induces tumor dormancy [74]. Non-invasive spheroids generally display collective/ epithelial migration. However, single- and mixed-cell migration here might mimic the initiation of metastasis. Heightened invasion index of H-5-Hyp and L-5-Hyp compared to L-0 condition reveals the role of hypoxia as a driving force behind the migration. The diminished level of MMP secretion in those conditions (L-0, H-5-Hyp, and L-5-Hyp) emphasizes the amoeboid migration modality, which was in line with a previous report [12]. Differential inhibitor assay shows that myosin II or protein kinase $\mathrm{C}$ mediated mode of amoeboid migration is not happening here [75] but MLC phosphorylation and MLCK mediated amoeboid migration is occurring [76]. This result can conclude that metabolically stressed spheroids, when rescued, choose MLCK mediated amoeboid migration as an escape route. This is in line with an earlier report of hypoxia-induced epithelial to amoeboid transition (EAT) [13]. After the rescue, invasive spheroids exhibit heightened sprouting/strand formation compared to control. They also show ample singe-cell migration within the collagen gel. We assume that both non-invasive and invasive stressed spheroids become more aggressive after the rescue, which parallels post-angiogenesis tumor aggression.

Tumor growth and proliferation depend on the stromal cell population, which might heal the metabolically challenged tumors $[77,78]$. Direct co-culture assay exhibits the successful recruitment of the mesenchymal stem cells into the stressed tumor. Compared to control, the stressed ones (L-5-Hyp and L-0-Hyp) are found to control the recruitment of stem cells more efficiently. The incorporation of stem cells within the stressed spheroids seems proportionate to their nutritional need. It is clear from this study that metabolically challenged tumors can influence and use stem cells for metabolical support.

It is observed here and supported by numerous other reports that individual stress conditions alone, such as hypoxia, are not enough to introduce de novo capability for organ colonization [13]. This study highlights that not a single one but the incorporation of multiple stress conditions may re-engineer the nature of a spheroid into a more aggressive one, irrespective of its previous status. This study also emphasizes the long-term implications of metabolic stress on tumor survival through initiating the dormant stage and enhanced recruitment of stromal cells. Such metabolically challenged spheroids can further serve as a platform for studying the synergistic effect of fasting and chemotherapy [79]. Furthermore, the connection between the macro- and micro-nutrients and in vitro tumorigenesis can be utilized in the future to ascertain the molecular mechanism behind low BMI and minimal cancer risk in real life.

\section{Conclusion}

Though genetic and epigenetic changes at the global range play the most crucial role in triggering the metastatic cascade of cancer, local environmental stress might play a critical role too. Tumor cells can re-engineer their metabolic pathways to resist or accept local micro-environmental changes such as hypoxia or nutritional stress. Additionally, the adverse situations influence the phenotype of the tumor and might nudge the plastic tumor cells towards the aggressive end of the spectrum. Stress conditions-mediated recruitment of stromal cells can also replenish the heterogeneous nature of the tumor. In the future, such a platform could be helpful to study the effect of the metabolical altercation on tumor phenotype and behavior in detail.

\section{Limitation of this study}

This study tried to recapitulate the multiplex nutritional stress conditions a growing tumor faces within a 
minimalistic in vitro platform. However, the static nature of the experiments is not comparable to a dynamic physiological condition. Additionally, other components of a growing tumor, namely CAFs, immune cells, and others, are not present in this model, which might recompense the effect of the stress in diverse ways. Such limitations can be remedied through a tumor on-chip model in the future.

Supplementary Information The online version contains supplementary material available at https://doi.org/10.1007/s44164-021-00006-5.

Acknowledgements T.D thanks UGC for the UGC-FRP scheme, and DST SERB and DBT for research funding. S.G thanks DST SERB and CSIR, India, for the fellowship. A.T thanks DBT and CSIR, India, for the fellowship. A.N thanks UPE II for the fellowship. The authors thank IBB, SPPU, and UPE II for infrastructure, central research facilities, and funding, respectively.

Author contribution TD and SG designed the experiments. SG performed the experiment with the help from AT and AN. TD and SG analyzed the data and wrote the manuscript.

\section{Declarations}

Conflict of interest The authors declare no competing interests.

\section{References}

1. Foulkes WD. Size surprise? Tumour size, nodal status, and outcome after breast cancer. Curr. Oncol. 2012;19:241-3. https:// doi.org/10.3747/co.19.1185.

2. Ben-Jacob E, Coffey DS, Levine H. Bacterial survival strategies suggest rethinking cancer cooperativity. Trends Microbiol. 2012;20(9):403-10. https://doi.org/10.1016/j.tim.2012.06.001.

3. Lorusso G, Rüegg C. The tumor microenvironment and its contribution to tumor evolution toward metastasis. Histochem Cell Biol. 2008;130(6):1091-103. https://doi.org/10.1007/ s00418-008-0530-8.

4. Lu P, Weaver VM, Werb Z. The extracellular matrix: a dynamic niche in cancer progression. J Cell Biol. 2012;196(4):395-406. https://doi.org/10.1083/jcb.201102147.

5. Flemming A. Stress can fuel tumour angiogenesis. Nat Rev Drug Discov. 2006;5(9):721-1. https://doi.org/10.1038/nrd21 41.

6. Senft D, Ronai ZA. Adaptive stress responses during tumor metastasis and dormancy. Trends in Cancer. 2016;2(8):429-42. https://doi.org/10.1016/j.trecan.2016.06.004.

7. Javier G-B, Williams RT, Guarecuco R, Birsoy K. Targeting extracellular nutrient dependencies of cancer cells. Molecular Metabolism. 2020;33(2):67-82. https://doi.org/10.1016/j.molmet.2019.11.011.

8. Wu H, Ding Z, Hu D, et al. Central role of lactic acidosis in cancer cell resistance to glucose deprivation-induced cell death. J Pathol. 2012;227(2):189-99. https://doi.org/10.1002/path.3978.

9. Spitz DR, Je S, La R, Galoforo SS, Lee YJ. Glucose deprivationinduced oxidative stress in human tumor cells: a fundamental defect in metabolism? Ann N Y Acad Sci. 2006;899(1):349-62. https://doi.org/10.1111/j.1749-6632.2000.tb06199.x.
10. Ye J, Kumanova M, Hart LS, et al. The GCN2-ATF4 pathway is critical for tumour cell survival and proliferation in response to nutrient deprivation. EMBO J. 2010;29(12):2082-96. https:// doi.org/10.1038/emboj.2010.81.

11. Wang Y, Ning Y, Alam GN, et al. Amino acid deprivation promotes tumor angiogenesis through the GCN2/ATF4 pathway. Neoplasia. 2013;15(8):989-97. https://doi.org/10.1593/neo. 13262

12. Geis T, Döring C, Popp R, et al. HIF-2alpha-dependent PAI-1 induction contributes to angiogenesis in hepatocellular carcinoma. Exp Cell Res. 2015;331:46-57. https://doi.org/10.1016/j. yexcr.2014.11.018.

13. Lehmann S, te Boekhorst V, Odenthal J, et al. Hypoxia induces a HIF-1-dependent transition from collective-toamoeboid dissemination in epithelial cancer cells. Curr Biol. 2017;27(3):392-400. https://doi.org/10.1016/j.cub.2016.11.057.

14. Bielenberg DR, Zetter BR. The contribution of angiogenesis to the process of metastasis. Cancer J. 2015;21(4):267-73. https:// doi.org/10.1097/PPO.0000000000000138.

15. Folkman J. Angiogenesis inhibitors: a new class of drugs. Cancer Biol Ther. 2003;2:S127-33. https://doi.org/10.4161/cbt.212.

16. Wang D, Tan C, Xiao F, et al. The "inherent vice" in the antiangiogenic theory may cause the highly metastatic cancer to spread more aggressively. Sci Rep. 2017a;7:2365. https://doi. org/10.1038/s41598-017-02534-1.

17. Zeng Y, Yao X, Liu X, et al. Anti-angiogenesis triggers exosomes release from endothelial cells to promote tumor vasculogenesis. J Extracell Vesicles. 2019;8:1629865. https://doi. org/10.1080/20013078.2019.1629865.

18. Laderoute KR, Amin K, Calaoagan JM, et al. 5'-AMP-activated protein kinase (AMPK) is induced by low-oxygen and glucose deprivation conditions found in solid-tumor microenvironments. Mol Cell Biol. 2006;26(14):5336-47. https://doi.org/10.1128/ MCB.00166-06.

19. Scott L, Lamb J, Smith S, Wheatley DN. Single amino acid (arginine) deprivation: rapid and selective death of cultured transformed and malignant cells. Br J Cancer. 2000;83(6):800 10. https://doi.org/10.1054/bjoc.2000.1353.

20. Eales KL, Hollinshead KER, Tennant DA. Hypoxia and metabolic adaptation of cancer cells. Oncogenesis. 2016;5(1):e190. https://doi.org/10.1038/oncsis.2015.50.

21. Wang $\mathrm{P}$, Long $\mathrm{M}$, Zhang $\mathrm{S}$, et al. Hypoxia-inducible factor- $1 \alpha$ regulates autophagy via the $\mathrm{p} 27-\mathrm{E} 2 \mathrm{~F} 1$ signaling pathway. Mol Med Rep. 2017;16(2):2107-12. https://doi.org/10.3892/mmr. 2017.6794.

22. Carmeliet P, Dor Y, Herbert JM, et al. Role of HIF-1alpha in hypoxia-mediated apoptosis, cell proliferation and tumour angiogenesis. Nature. 1998;394(6692):485-90. https://doi.org/ $10.1038 / 28867$

23. Schroll MM, Liu X, Herzog SK, Skube SB, Hummon AB. Nutrient restriction of glucose or serum results in similar proteomic expression changes in 3D colon cancer cell cultures. Nutr Res. 2016;36(10):1068-80. https://doi.org/10.1016/j.nutres.2016.08. 002.

24. Osawa T, Muramatsu M, Watanabe M, Shibuya M. Hypoxia and low-nutrition double stress induces aggressiveness in a murine model of melanoma. Cancer Sci. 2009;100(5):844-51. https:// doi.org/10.1111/j.1349-7006.2009.01105.x.

25. Caino MC, Chae YC, Vaira V, et al. Metabolic stress control of cytoskeletal dynamics and metastasis. J Clin Invest. 2013;123(7):2907-20. https://doi.org/10.1172/JCI67841DS1.

26. Lee E, Yang $\mathrm{J}, \mathrm{Ku} \mathrm{M}$, et al. Metabolic stress induces a Wntdependent cancer stem cell-like state transition. Cell Death Dis. 2015;6(7):e1805. https://doi.org/10.1038/cddis.2015.171.

27. Aftab S, Shakoori AR. Low glucose availability alters the expression of genes involved in initial adhesion of human 
glioblastoma cancer cell line SF767. J Cell Biochem. 2019;120(10):16824-39. https://doi.org/10.1002/jcb.28940.

28. Wek RC, Staschke KA. How do tumours adapt to nutrient stress? EMBO J. 2010;29(12):1946-7. https://doi.org/10.1038/ emboj.2010.110.

29. Izuishi K, Kato K, Ogura T, Kinoshita T, Esumi H. Remarkable tolerance of tumor cells to nutrient deprivation: possible new biochemical target for cancer therapy. Cancer Res. 2000;60(21):6201-7.

30. Katt ME, Placone AL, Wong AD, Xu ZS, Searson PC. In vitro tumor models: advantages, disadvantages, variables, and selecting the right platform. Front Bioeng Biotechnol. 2016;4:1-14. https://doi.org/10.3389/fbioe.2016.00012.

31. Sontheimer-Phelps A, Hassell BA, Ingber DE. Modelling cancer in microfluidic human organs-on-chips. Nat Rev Cancer. 2019;19(2):65-81. https://doi.org/10.1038/s41568-018-0104-6.

32. Zang Q, Sun C, Chu X, Li L, Gan W, Zhao Z, et al. Spatially resolved metabolomics combined with multicellular tumor spheroids to discover cancer tissue relevant metabolic signatures. Anal Chim Acta. 2021;1155:338342. https://doi.org/10. 1016/j.aca.2021.338342.

33. Tomar G, Srivastava RK, Gupta N, Barhanpurkar AP, Pote ST, Jhaveri HM, et al. Human gingiva-derived mesenchymal stem cells are superior to bone marrow-derived mesenchymal stem cells for cell therapy in regenerative medicine. Biochem Biophys Res Commun. 2010;393(3):377-83. https://doi.org/10. 1016/j.bbrc.2010.01.126.

34. Freischel AR, Damaghi, Cunningham JJ, Hashim AI, Gillies RJ, Gatenby RA, Brown JS. Frequency-dependent interactions determine outcome of competition between two breast cancer cell lines. Sci Rep. 2021;11:4908. https://doi.org/10.1038/ s41598-021-84406-3.

35. Borowicz S, Scoyk MV, Avasarala S, et al. The Soft Agar Colony Formation Assay. J. Vis. Exp. 2014;(92):e51998. https:// doi.org/10.3791/51998

36. Gayan S, Teli A, Dey T. Inherent aggressive character of invasive and non-invasive cells dictates the in vitro migration pattern of multicellular spheroid. Sci Rep. 2017;7(1):11527. https://doi.org/10.1038/s41598-017-10078-7.

37. Clémence D, Robin D, Pierre D, Corinne A, Claire S, Christelle B, Emmanuelle M, Frédérique PL, Bamdad M. Development and cytotoxic response of two proliferative MDA-MB-231 and non-proliferative SUM1315 three-dimensional cell culture models of triple-negative basal-like breast cancer cell lines. Oncotarget. 2017;8(56):95316-31. https://doi.org/10.18632/oncot arget.20517.

38. Kumar R, Saha S, Sinha B. Cell spread area and traction forces determine myosin-II-based cortex thickness regulation. Biochim Biophys Acta Mol Cell Res. 2019;1866(12):118516. https://doi. org/10.1016/j.bbamcr.2019.07.011.

39. Manuel Iglesias J, Beloqui I, Garcia-Garcia F, et al. Mammosphere formation in breast carcinoma cell lines depends upon expression of E-cadherin. Gotoh N, ed. PLoS One. 2013;8(10):e77281. 10.1371/journal.pone.0077281.

40. Ivascu A, Kubbies M. Diversity of cell-mediated adhesions in breast cancer spheroids. Int J Cancer. 2007;31:1403-13 http:// www.nature.com/articles/s41598-020-58124-1.

41. Lin R-Z, Chou L-F, Chien C-CM, Chang H-Y. Dynamic analysis of hepatoma spheroid formation: roles of E-cadherin and $\beta 1$-integrin. Cell Tissue Res. 2006;324(3):411-22. https://doi. org/10.1007/s00441-005-0148-2.

42. Kim S-J, Kim EM, Yamamoto M, Park H, Shin H. Engineering multi-cellular spheroids for tissue engineering and regenerative medicine. Adv Healthc Mater. 2020;9(23):e2000608. https://doi. org/10.1002/adhm.202000608.
43. Wang H, Paczulla A.M, Konantz M, and Lengerke C. In vitro tumorigenic assay: the tumor spheres assay. Cancer stem cells: methods and protocols, Methods in Molecular Biology. 2018;1692: 77-87. doi: https://doi.org/10.1007/978-1-4939-7401-6_7.

44. Kronemberger GS , Miranda G. A. S. C, , Tavares R.S.N, Montenegro B, de A. Kopke Ú and Baptista L.S. Recapitulating tumorigenesis in vitro: opportunities and challenges of $3 \mathrm{D}$ bioprinting. Front Bioeng Biotechnol. 2012;9: 428. 10.3389/ fbioe. 2021.682498

45. Jo H, Lee J, Jeon J, et al. The critical role of glucose deprivation in epithelial-mesenchymal transition in hepatocellular carcinoma under hypoxia. Sci Rep. 2020;10(1):1538. https://doi.org/10.1038/ s41598-020-58124-1.

46. Ross E, Ata R, Thavarajah T, et al. AMP-activated protein kinase regulates the cell surface proteome and integrin membrane traffic. Caplan S, ed. PLoS One. 2015;10(5):e0128013. 10.1371/journal. pone.0128013.

47. Jones JC, Miceli AM, Chaudhry MM, et al. Glucose-limiting conditions induce an invasive population of MDA-MB-231 breast cancer cells with increased connexin 43 expression and membrane localization. J Cell Commun Signal. 2021;15(2):223-36. https:// doi.org/10.1007/s12079-020-00601-3.

48. Anderson ARA, Weaver AM, Cummings PT, Quaranta V. Tumor morphology and phenotypic evolution driven by selective pressure from the microenvironment. Cell. 2006;127(5):905-15. https:// doi.org/10.1016/j.cell.2006.09.042.

49. Roy M, Finley SD. Metabolic reprogramming dynamics in tumor spheroids: Insights from a multicellular, multiscale model. Maini PK, editor. PLOS Comput Biol. 2019;15: e1007053. 10.1371/ journal.pcbi.1007053

50. Sarrió D, Palacios J, Hergueta-Redondo M, Gómez-López G, Cano A, Moreno-Bueno G. Functional characterization of Eand $\mathrm{P}$-cadherin in invasive breast cancer cells. BMC Cancer. 2009;9:74. https://doi.org/10.1186/1471-2407-9-74.

51. Nass SJ, Herman JG, Gabrielson E, Iversen PW, Parl FF, Davidson NE, Graff JR. Aberrant methylation of the estrogen receptor and E-cadherin $5 \mathrm{CpG}$ islands increases with malignant progression in human breast cancer. Cancer Res. 2000;60:4346-8.

52. Jalaguier-Coudray A, Thomassin-Piana J. Solid masses: what are the underlying histopathological lesions? Diagn. Interv. Imaging. 2014;95:153-68. https://doi.org/10.1016/j.diii.2013.12.014.

53. Larribe M, Thomassin-Piana J, Jalaguier-Coudray A. Breast cancers with round lumps: correlations between imaging and anatomopathology. Diagn Interv Imaging. 2014;95(1):37-46. https:// doi.org/10.1016/j.diii.2013.04.003.

54. Goyal U, Ta M. A novel role of vitronectin in promoting survival of mesenchymal stem cells under serum deprivation stress. Stem Cell Res Ther. 2020;11(1):181. https://doi.org/10.1186/ s13287-020-01682-y.

55. Munir R, Lisec J, Swinnen JV, Zaidi N. Lipid metabolism in cancer cells under metabolic stress. Br J Cancer. 2019;120(12):10908. https://doi.org/10.1038/s41416-019-0451-4.

56. Adler JJ, Johnson DE, Heller BL, et al. Serum deprivation inhibits the transcriptional co-activator YAP and cell growth via phosphorylation of the 130-kDa isoform of Angiomotin by the LATS1/2 protein kinases. Proc Natl Acad Sci U S A. 2013;110(43):1736873. https://doi.org/10.1073/pnas.1308236110.

57. Palorini R, Cammarata FP, Cammarata F, et al. Glucose starvation induces cell death in K-ras-transformed cells by interfering with the hexosamine biosynthesis pathway and activating the unfolded protein response. Cell Death Dis. 2013;4(7):e732. https://doi.org/ 10.1038/cddis.2013.257.

58. Chen X, Meng Q, Zhao Y, et al. Angiotensin II type 1 receptor antagonists inhibit cell proliferation and angiogenesis in breast cancer. Cancer Lett. 2013;328(2):318-24. https://doi.org/10. 1016/j.canlet.2012.10.006. 
59. Iurlaro R, Püschel F, León-Annicchiarico CL, et al. Glucose deprivation induces ATF4-mediated apoptosis through TRAIL death receptors. Mol Cell Biol. 2017;37(10). 10.1128/MCB.00479-16.

60. Nagarajah NS, Vigneswaran N, Zacharias W. Hypoxia-mediated apoptosis in oral carcinoma cells occurs via two independent pathways. Mol Cancer. 2004;3(1):38. https://doi.org/10.1186/ 1476-4598-3-38.

61. Kim JW, Ho WJ, Wu BM. The role of the $3 \mathrm{D}$ environment in hypoxia-induced drug and apoptosis resistance. Anticancer Res. 2011;31(10):3237-46.

62. Stöhr D, Schmid JO, Beigl TB, Mack A, Maichl DS, Cao K, et al. Stress-induced TRAILR2 expression overcomes TRAIL resistance in cancer cell spheroids. Cell Death Differ. 2020;27:303752. https://doi.org/10.1038/s41418-020-0559-3.

63. Dikovskaya D, Cole JJ, Mason SM, et al. Mitotic stress is an integral part of the oncogene-induced senescence program that promotes multinucleation and cell cycle arrest. Cell Rep. 2015;12(9):1483-96. https://doi.org/10.1016/j.celrep.2015.07. 055.

64. Krajcovic. A non-genetic route to aneuploidy in human cancers. Nat Cell Biol. 2011;175(1):88-99. https://doi.org/10.1001/jamai nternmed.2014.4010.Influence.

65. Hamann JC, Surcel A, Chen R, et al. Entosis is induced by glucose starvation. Cell Rep. 2017;20(1):201-10. https://doi.org/10. 1016/j.celrep.2017.06.037.

66. Papandreou ME, Tavernarakis N. Nucleophagy: from homeostasis to disease. Cell Death Differ. 2019;26:630-9. https://doi.org/10. 1038/s41418-018-0266-5.

67. Ziperstein J.M, Guzman A, Kaufman L.J. Breast cancer cell line aggregate morphology does not predict invasive capacity. PLoS ONE. 2015; 10(9): e0139523. doi:https://doi.org/10.1371/journ al.pone.0139523.

68. Clark AG, Dierkes K, Paluch EK. Monitoring actin cortex thickness in live cells. Biophys J. 2013;105(3):570-80. https://doi.org/ 10.1016/j.bpj.2013.05.057.

69. Tavares S, Vieira AF, Taubenberger AV, et al. Actin stress fiber organization promotes cell stiffening and proliferation of pre-invasive breast cancer cells. Nat Commun. 2017;8(1):15237. https:// doi.org/10.1038/ncomms 15237 .
70. Recouvreux MV, Commisso C. Macropinocytosis: a metabolic adaptation to nutrient stress in cancer. Front Endocrinol. 2017;8:1-7. https://doi.org/10.3389/fendo.2017.00261.

71. Sosa MS, Bragado P, Aguirre-Ghiso JA. Mechanisms of disseminated cancer cell dormancy: an awakening field. Nat Rev Cancer. 2014;14(9):611-22. https://doi.org/10.1038/nrc3793.

72. Kechagia JZ, Ivaska J, Roca-Cusachs P. Integrins as biomechanical sensors of the microenvironment. Nat Rev Mol Cell Biol. 2019;20(8):457-73. https://doi.org/10.1038/s41580-019-0134-2.

73. Lu Z, Xie J, Wu G, et al. Fasting selectively blocks development of acute lymphoblastic leukemia via leptin-receptor upregulation. Nat Med. 2017;23(1):79-90. https://doi.org/10.1038/nm.4252.

74. Barney LE, Hall CL, Schwartz AD, et al. Tumor cell-organized fibronectin maintenance of a dormant breast cancer population. Sci Adv. 2020;6(11):eaaz4157. https://doi.org/10.1126/sciadv. aaz4157.

75. Lämmermann T, Sixt M. Mechanical modes of "amoeboid" cell migration. Curr Opin Cell Biol. 2009;21(5):636-44. https://doi. org/10.1016/j.ceb.2009.05.003.

76. Chen $\mathrm{C}$, Tao $\mathrm{T}$, Wen $\mathrm{C}$, et al. Myosin light chain kinase (MLCK) regulates cell migration in a myosin regulatory light chain phosphorylation-independent mechanism. J Biol Chem. 2014;289(41):28478-88. https://doi.org/10.1074/jbc.M114. 567446.

77. Jung Y, Kim JK, Shiozawa Y, et al. Recruitment of mesenchymal stem cells into prostate tumours promotes metastasis. Nat Commun. 2013;4(1):1795. https://doi.org/10.1038/ncomms2766.

78. Kalimuthu S, Oh JM, Gangadaran P, Zhu L, Lee HW, Rajendran RL, et al. In Vivo Tracking of chemokine receptor CXCR4-engineered mesenchymal stem cell migration by optical molecular imaging. Stem Cells Int. 2017;2017:8085637. https://doi.org/10. 1155/2017/8085637.

79. Lee C, Raffaghello L, Brandhorst S, et al. Fasting cycles retard growth of tumors and sensitize a range of cancer cell types to chemotherapy. Sci Transl Med. 2012;4(124):124ra27. https://doi. org/10.1126/scitranslmed.3003293. 